Submitted to ... manuscript No.

(will be inserted by the editor)

\title{
GEOMETRY OF INTERACTIONS IN COMPLEX BODIES
}

\author{
Chiara de Fabritiis $^{1}$, Paolo Maria Mariano ${ }^{2}$ \\ 1 Dipartimento di Scienze Matematiche, \\ Università Politecnica delle Marche, \\ via Brecce Bianche, Ancona (Italy) \\ e-mail: fabritii@dipmat.univpm.it \\ 2 Dipartimento di Ingegneria Strutturale e Geotecnica, \\ Università di Roma "La Sapienza", \\ via Eudossiana 18, I-00184 Roma (Italy) \\ e-mail: paolo.mariano@uniroma1.it, \\ now at Università del Molise, Campobasso (Italy).
}

The date of receipt and acceptance will be inserted by the editor

\begin{abstract}
We analyze geometrical structures necessary to represent bulk and surface interactions of standard and substructural nature in complex bodies. Our attention is mainly focused on the influence of diffuse interfaces on sharp discontinuity surfaces. In analyzing this phenomenon, we prove the covariance of surface balances of standard and substructural interactions.
\end{abstract}

Key words Multifield theories, complex materials, discontinuity surfaces, Lie symmetries

\section{Introduction}

Bodies are called complex when their material substructure (i.e. the texture from nano-level to meso-level) has a prominent influence on their gross behavior. Such an influence appears also through a not negligible occurrence of interactions due to substructural changes. Examples are liquid crystals, elastomers, ferroelectric and microcracked bodies, spin glasses. Above all, soft condensed matter displays complex behavior. Applications in nanotechnology, smart structures and various fields of technology open basic theoretical and experimental problems that challenge, in a certain sense, even some aspects of the foundational concepts of standard continuum mechanics. Basically, the standard paradigm of Cauchy's format of continuum mechanics, prescribing that the material element is a sort of indistinct sphere that we 
collapse in a point in space seems to be not sufficient to account for the articulated substructural nature of a complex body.

In fact, for complex bodies the material element is rather a 'system' and one needs the introduction of appropriate morphological descriptors $\boldsymbol{\nu}$ of such a system (order parameters) at least at a coarse grained level. They describe the essential geometrical features of substructural shapes.

Physical circumstances of disparate nature suggest a rich crop of possible choices of $\boldsymbol{\nu}$, each one characterizing special models. Moreover, the selection of morphological descriptors is strongly related with the representation of substructural interactions arising within each material element and between neighboring material elements as a consequence of substructural changes. Interactions are represented in fact by objects conjugated in the sense of power with the rates of the quantities describing the geometry of the body and its changes. In this sense, since placement and order parameter fields are involved, the description of complex bodies adopted here is called multifield. It has basic differences with standard internal variable models. In a multifield approach, morphological descriptors enter directly the geometrical representation of the body and its kinematics; true interactions are associated with their rates and balanced. Information about the material substructure are introduced already at the level of geometrical description of the body. On the contrary, in standard internal variable models, the geometrical description of the body is of Cauchy's type: the material element is morphologically equivalent to an indistinct sphere described just by its place in space. There, internal variables come into play in constructing thermodynamics to carry information about the material substructure just at constitutive level (a detailed treatment of these classes of models can be found in [34]).

By following the general unified framework of multifield theories proposed by Capriz in 1989 [4] (see also [3]) and then developed further on in its abstract structure (see [32], [23], [8], [9]), we do not specify the nature of $\boldsymbol{\nu}$. We require only that $\boldsymbol{\nu}$ be an element of a finite-dimensional differentiable paracompact manifold without boundary $\mathcal{M}$ to cover a class as large as possible of special theories. Our attention is then focused on the general setting which contains as special cases prominent theories interpreting problems typical of condensed matter. In a certain sense our work deals with a model of models. However, if we tackle this general point of view, we face the basic difficulty that $\mathcal{M}$ does not coincide with a linear space in general. Moreover, we cannot consider a priori $\mathcal{M}$ embedded in a linear space because the embedding is a non-trivial part of some special modeling.

We focus our attention on conservative processes. For them, the relevant appropriate Hamiltonian formalism has been developed in [9] as an evolution of the Hamiltonian formalism in classical non-linear elasticity (the one discussed in [27]). We start from the results in [9] and analyze some of the rather subtle geometrical questions induced by the abstract nature of $\mathcal{M}$.

Our essential point of view is the one of [25]: "Geometry and mechanics of maps between manifolds are a general framework for condensed matter 
physics and are also a tool to construct new models of unusual and perhaps unaspected phenomena."

The main attention is focused on the representation of the way in which diffuse interfaces influence the behavior of additional sharp interfaces. In fact, the presence of the gradient of $\boldsymbol{\nu}$ in the list of entries of the energy may account for diffuse interfaces as well as it may describe weakly nonlocal (i.e. gradient) effects in homophase materials. Additional discontinuity surfaces may also occur as a consequence of the presence of defects, shock or acceleration waves, solidification phenomena of complex fluids etc. As an example one may consider a polarized ferroelectric material in which external loads induce a shock wave: the shock front encounters walls of polarized domains and interact with them. So, in general, one may say that, in principle, diffuse interfaces and potential homophase gradient effects may interact with sharp discontinuity surfaces influencing their possible evolution. In particular, when discontinuity surfaces are structured, in the sense that they are endowed with surface energy, surface interactions may occur. They are of standard and substructural nature. Standard surface stresses are in fact due to the deformation of the discontinuity surface while the other are a consequence of changes of substructure at the surface itself.

Surface balances involving standard surface stresses have been obtained in [17] while substructural measures of interactions have been introduced in [22], [23] and the relevant balance equations derived there (see also [8]). However, there is no proof of their covariance. Such a proof is provided here (see Theorem 2) and is the main result of this paper. In obtaining it we enlarge the standard notion of observer: for us, in fact, such a notion involves not only the representation of the ambient space and the time scale, but also the representation of the manifold of substructural morphologies (see also [25]).

\section{Morphology of complex bodies}

We consider a body occupying a regular region $\mathcal{B}_{0}$ of the three-dimensional Euclidean space $\mathcal{E}^{3}$ (with affine translation space $\left.V e c\right)^{1}$. The current morphology of the body is described by two sufficiently smooth mappings:

$$
\mathcal{B}_{0} \ni \mathbf{X} \stackrel{\tilde{\mathbf{x}}}{\longmapsto} \mathbf{x}=\tilde{\mathbf{x}}(\mathbf{X}) \in \mathcal{E}^{3} \quad, \quad \mathcal{B}_{0} \ni \mathbf{X} \stackrel{\tilde{\boldsymbol{\nu}}}{\longmapsto} \boldsymbol{\nu}=\tilde{\boldsymbol{\nu}}(\mathbf{X}) \in \mathcal{M} .
$$

1. $\tilde{\mathbf{x}}$ shows the current placement of a material element at $\mathbf{X}$ in $\mathcal{B}_{0}$, is injective and orientation preserving, and $\mathcal{B}=\mathbf{x}\left(\mathcal{B}_{0}\right)$ is also regular as $\mathcal{B}_{0}$.

\footnotetext{
1 With the adjective 'regular' we refer to an open (bounded) subset of $\mathcal{E}^{3}$ coinciding with the interior of its closure, with a surface-like boundary where the outward unit normal $\mathbf{n}$ is well-defined everywhere to within a finite number of corners and edges. The treatment of infinite bodies requires only some minor technical adjustments in the results of the present paper. In any case, some remarks about them are presented throughout the paper.
} 
2. $\tilde{\boldsymbol{\nu}}$ is the order parameter map and assigns to each material element a coarse grained morphological descriptor $\tilde{\boldsymbol{\nu}}(\mathbf{X})$ of its substructure (order parameter), chosen as an element of a differentiable paracompact manifold $\mathcal{M}$ (generally without boundary).

Two natural tangent maps arise, namely $T \mathbf{x}: T \mathcal{B}_{0} \rightarrow T \mathcal{B}$ and $T \boldsymbol{\nu}$ : $T \mathcal{B}_{0} \rightarrow T \mathcal{M}$. The pairs $(\mathbf{x}, \nabla \mathbf{x}),(\boldsymbol{\nu}, \nabla \boldsymbol{\nu})$ are the peculiar elements of $T \mathbf{x}$ and $T \boldsymbol{\nu}$ respectively. Since $T \mathcal{B}_{0}$ is a trivial bundle and a connection is natural over it, we can separate in invariant way $\mathbf{x}$ from $\nabla \mathbf{x}$ which is commonly indicated with $\mathbf{F}$. The condition that $\tilde{\mathbf{x}}$ be orientation preserving implies that, at each $\mathbf{X} \in \mathcal{B}_{0}, \mathbf{F} \in \operatorname{Hom}\left(T_{\mathbf{X}} \mathcal{B}_{0}, T_{\tilde{\mathbf{x}}(\mathbf{X})} \mathcal{B}\right)$ has positive determinant. Since $\mathcal{M}$ is a priori not trivial, the pair $(\boldsymbol{\nu}, \nabla \boldsymbol{\nu})$ cannot be separated in invariant way, unless there is a parallelism over $\mathcal{M}$. In principle, one may define in abstract way a parallelism over $\mathcal{M}$ but, since the pair $(\boldsymbol{\nu}, \nabla \boldsymbol{\nu})$ enters constitutive issues, one should have a physically significant parallelism. In other words, when we act separating in invariant way $\nabla \boldsymbol{\nu}$ from $\boldsymbol{\nu}$ we should presume to have at least one physically significant parallelism over $\mathcal{M}$ even when circumstances may allow us the use of invariance requirements with respect to the choice of the connection. In fact, the presence of the pair $(\boldsymbol{\nu}, \nabla \boldsymbol{\nu})$ or of arbitrarily one element of it (namely $\boldsymbol{\nu}$ or $\nabla \boldsymbol{\nu})$ in the list of entries of the energy changes the representation of interactions.

As remarked in the introduction, in dealing with the geometrical (morphological) description of complex bodies, we relax one of the axioms of the mechanics of simple materials. In our picture, in fact, the material element is not morphologically equivalent to a 'monad', a simple material particle in the sense of Cauchy (see [29], [30]), identified only by its place in space. Instead, we consider the body as a collection of subsystems of the same nature (the material substructure) and the order parameter at a given point represents the characteristic features of the morphology of the subsystem there.

Sometimes the material substructure is a perfectly identifiable Lagrangian system as in the case of nematic liquid crystals [14], [20], [11] (in which the nematic molecules can be separated from the melt), sometimes it does not as in granular gases [6] and microcracked bodies [26]. In granular gases, e.g., a material element collects a family of sparse granules with peculiar velocities, so that the order parameter may be an element of a suitable Grassmanian of the tangent bundle of some finite-dimensional manifold, while for microcracked bodies each microcrack can be considered either as a sharp planar defect not interpenetrated by interatomic bonds or as an elliptic void, so it does not exist per se and has a volatile substance determined just by the surrounding matter.

We leave undetermined the specific nature of $\boldsymbol{\nu}$ to cover a class as large as possible of special cases, following in this way the unifying point of view of Capriz [4]. Our primary strategy is to work with minimal requirements for $\mathcal{M}$ and to add geometrical structure to it only when necessary. 
In this way we avoid the path leading to a monotonous zoo of special models with slightly different formal aspects but with the same intrinsic nature.

With respect to Hamiltonian elasticity of simple bodies (see [27], Chap. 4 ), here the main source of difficulties is the circumstance that $\mathcal{M}$ is a nontrivial manifold; in particular it does not coincide with a linear space in most cases of prominent interest.

The space $\mathcal{C}$ of pairs of maps $(\tilde{\mathbf{x}}, \tilde{\boldsymbol{\nu}})$, a product space of the type $\mathcal{C}_{\mathbf{x}} \times \mathcal{C}_{\boldsymbol{\nu}}$, with $\tilde{\mathbf{x}}$ pertaining to $\mathcal{C}_{\mathbf{x}}$ and $\tilde{\boldsymbol{\nu}}$ to $\mathcal{C}_{\boldsymbol{\nu}}$, has a non-trivial structure which depends on the geometrical properties of $\mathcal{M}$. Basically, we imagine that $\mathcal{C}_{\mathbf{x}} \subseteq$ $W^{1, p}\left(\mathcal{B}_{0}, V e c\right)$ for some $p \geq 1$ and $\mathcal{C}_{\boldsymbol{\nu}}=P C^{1}\left(\mathcal{B}_{0}, \mathcal{M}\right)$, i.e. we require that the order parameter map be at least continuous and piecewise continuously differentiable over $\mathcal{B}_{0}$, while the placement map be an element of the Sobolev space $W^{1, p}\left(\mathcal{B}_{0}, V e c\right)$, even if we may basically require that $\tilde{\mathbf{x}}$ be continuous and piecewise continuously differentiable too.

Specific examples showing the possible intricate nature of $\mathcal{C}$, in particular of $\mathcal{C}_{\boldsymbol{\nu}}$, can be discussed at length.

We analyze below the case in which $\mathcal{M}$ has a Riemannian structure. However, the developments in subsequent sections do not require strictly such a structure that will be called upon only when necessary.

In any case, even when $\mathcal{M}$ is Riemannian, we do not assign a priori any prevalent rôle to Levi-Civita connection.

2.1 The case in which $\mathcal{M}$ has a Riemannian structure: aspects of the nature of $\mathcal{C}$

We assume just in this section that $\mathcal{M}$ is a Riemannian, connected, finite dimensional manifold, endowed with a metric $\mathbf{g}_{\mathcal{M}}$ and a consequent LeviCivita connection.

Notice that we do not require here the embedding of $\mathcal{M}$ in some linear space. When, in fact, we embed $\mathcal{M}$ in a linear space by using, let say, Nash's isometric embedding to preserve at least he quadratic part of the kinetic energy (if it exists) pertaining to the substructure, the embedding is not unique. So the choice of it becomes matter of modeling. Moreover, the embedding is also not strictly necessary to build up the structures needed for the basic aspects of mechanics. For this reason, with the aim to eliminate overstructures as much as possible, we consider $\mathcal{M}$ in its abstract setting, not embedded a priori in a linear space.

We denote with $\langle\cdot, \cdot\rangle_{T \mathcal{M}}$ the scalar product over $T_{\boldsymbol{\nu}} \mathcal{M}$ associated with $\mathbf{g}_{\mathcal{M}}$. For any $C^{1}$-curve $\left[0, s^{*}\right] \ni s \longmapsto \boldsymbol{\nu}(s) \in \mathcal{M}$, we have a vector field $\boldsymbol{v}(s)=\frac{d \boldsymbol{\nu}}{d s}(s)$, and for any other vector field $A(s)$ over $\mathcal{M}$ one defines its derivative along $s \longmapsto \boldsymbol{\nu}(s)$ writing $\frac{D A}{d s}=\nabla_{\boldsymbol{v}(s)} A$, and call $A$ autoparallel along $s \longmapsto \boldsymbol{\nu}(s)$ when $\frac{D A}{d s}=0$ for any $s \in\left[0, s^{*}\right]$. As usual, $s \longmapsto \boldsymbol{\nu}(s)$ is a geodesic when $s \longmapsto \boldsymbol{v}(s)$ is autoparallel along it. 
The Riemannian structure assures the existence of a natural distance $d_{\mathcal{M}}: \mathcal{M} \times \mathcal{M} \rightarrow \mathbb{R}^{+}$over $\mathcal{M}$. In fact, by indicating with $\lambda$ any arbitrary piecewise $C^{1}$ curve $\lambda:\left[0, s^{*}\right] \rightarrow \mathcal{M}$ such that $\lambda(0)=\boldsymbol{\nu}_{1}$ and $\lambda\left(s^{*}\right)=\boldsymbol{\nu}_{2}$, as usual we put

$$
d_{\mathcal{M}}\left(\boldsymbol{\nu}_{1}, \boldsymbol{\nu}_{2}\right)=\inf \{l(\lambda)\}
$$

varying $\lambda$ between $\boldsymbol{\nu}_{1}$ and $\boldsymbol{\nu}_{2}$, with $l(\lambda)$ the length

$$
l(\lambda)=\int_{0}^{s^{*}}\left\langle\frac{d \lambda}{d s}(s), \frac{d \lambda}{d s}(s)\right\rangle_{T \mathcal{M}}^{\frac{1}{2}} d s
$$

(see, e.g., [1] Chapter 2, [33] Chapter 4 ). Locally, $d_{\mathcal{M}}$ is calculated over geodesics.

However, $d_{\mathcal{M}}$ may be unbounded. In this case, we select a new metric $\tilde{d}_{\mathcal{M}}: \mathcal{M} \times \mathcal{M} \rightarrow \mathbb{R}^{+}$over $\mathcal{M}$ defined by

$$
\tilde{d}_{\mathcal{M}}=\frac{d_{\mathcal{M}}}{1+d_{\mathcal{M}}}
$$

Notice that the metric $\tilde{d}_{\mathcal{M}}$

(a) is equivalent to $d_{\mathcal{M}}$ (it leads to the same topology),

(b) is bounded,

(c) is of class $C^{\infty}(\mathcal{M})$,

(d) if $d_{\mathcal{M}}$ is complete on $\mathcal{M}$, then $\tilde{d}_{\mathcal{M}}$ is complete too.

A well know theorem due to Hopf and Rinow ([19], p. 56) asserts that the following statements are equivalent: (i) $d_{\mathcal{M}}$ is complete, (ii) closed bounded sets with respect to $d_{\mathcal{M}}$ are compact, (iii) geodesics for $d_{\mathcal{M}}$ can be continued as to be defined on the whole real line. Moreover, if $\mathcal{M}$ is complete, the distance $d_{\mathcal{M}}$ can be computed over geodesics not only locally, but also globally.

Remark 1. When $\mathcal{M}$ is compact, $d_{\mathcal{M}}: \mathcal{M} \times \mathcal{M} \rightarrow \mathbb{R}^{+}$is bounded, then it is not necessary to substitute it with $\tilde{d}_{\mathcal{M}}$. Of course, the definition of $\tilde{d}_{\mathcal{M}}$ is just one of the possible ways to bound the metric (i.e., the 'diameter' of the manifold is finite).

By making use of $\tilde{d}_{\mathcal{M}}$ we may define three natural distances over $\mathcal{C}_{\boldsymbol{\nu}}$.

1. The first metric distance, indicated with $d^{(i)}$, is defined by

$$
d^{(i)}\left(\tilde{\boldsymbol{\nu}}_{1}, \tilde{\boldsymbol{\nu}}_{2}\right)=\int_{\mathcal{B}_{0}} \tilde{d}_{\mathcal{M}}\left(\boldsymbol{\nu}_{1}, \boldsymbol{\nu}_{2}\right) d(v o l),
$$

where $(i)$ stands for 'integral'.

2. Let $\left\{K_{n}\right\}$ be an exhaustion of $\mathcal{B}_{0}$, i.e. a compact cover of $\mathcal{B}_{0}$ such that

$K_{n} \subset \stackrel{\circ}{K}_{n+1}$ for any $n$. The second distance, indicated with $d^{(c)}$, is defined by

$$
d^{(c)}\left(\tilde{\boldsymbol{\nu}}_{1}, \tilde{\boldsymbol{\nu}}_{2}\right)=\sum_{n \in \mathbb{N}} \frac{1}{2^{-n}} \max _{\mathbf{X} \in K_{n}} \tilde{d}_{\mathcal{M}}\left(\boldsymbol{\nu}_{1}, \boldsymbol{\nu}_{2}\right),
$$

where $(c)$ stands for 'compact'. 
3. More simply, a third natural distance, indicated with $d^{(s)}$, can be defined by

$$
d^{(s)}\left(\tilde{\boldsymbol{\nu}}_{1}, \tilde{\boldsymbol{\nu}}_{2}\right)=\sup _{\mathbf{X} \in \mathcal{B}_{0}} \tilde{d}_{\mathcal{M}}\left(\boldsymbol{\nu}_{1}, \boldsymbol{\nu}_{2}\right)
$$

where $(s)$ stands for 'supremum'.

Remark 2. There is a peculiar physical difference between the metric $d^{(c)}$ and the other two. In fact, the exhaustion of $\mathcal{B}_{0}$ by compact sets, used to define $d^{(c)}$, implies that the values of the order parameter map over boundary points of $\mathcal{B}_{0}$ may contribute slightly to the distance in $\mathcal{C}_{\boldsymbol{\nu}}$, on the contrary of the other two metrics. In a certain sense, $d^{(c)}$ seems to be preferable when there are uncertainties in the physical meaning of boundary data about $\boldsymbol{\nu}$, a problem (the one of boundary data) that appears very subtle for some material substructures like microcracks.

Remark 3. Notice that $d^{(c)}$ and $d^{(s)}$ are complete over $C^{0}\left(\mathcal{B}_{0}, \mathcal{M}\right)$ if $\tilde{d}_{\mathcal{M}}$ is complete. In particular, compactness of $\mathcal{M}$ is sufficient for the validity of this statement.

Remark 4. We realize that the space of continuous maps between $\mathcal{B}_{0}$ and $\mathcal{M}$, namely $C\left(\mathcal{B}_{0}, \mathcal{M}\right)$, may be in general not complete with respect to the metric $d^{(i)}$. For example, let $\mathcal{B}_{0}$ coincide with the cube $(-1,2)^{3}$ in a frame $0 X_{1} X_{2} X_{3}$. We may analyze two different cases.

(i) Case 1: $\mathcal{M}$ is not compact. Let us assume $\mathcal{M} \equiv \mathbb{R}$, and for any $x, y \in \mathbb{R}$, put $d_{\mathcal{M}}(x, y)=|x-y|$. Let us construct also a sequence of $\boldsymbol{\nu}$ such that for $\left(X_{2}, X_{3}\right)$ ranging in $(-1,2)^{2}$

$$
\boldsymbol{\nu}_{n} \equiv f_{n}(\mathbf{X})=\left\{\begin{array}{cl}
0, & \text { if }-1<X_{1} \leq 0 \\
X_{1}^{n} & , \quad \text { if } 0 \leq X_{1} \leq 1 \\
1, & \text { if } 1 \leq X_{1}<2
\end{array}\right.
$$

From (5), for $n \leq m \in \mathbb{N}$, we get

$$
\begin{aligned}
d^{(i)}\left(f_{n}, f_{m}\right) & =\int_{\mathcal{B}_{0}} \tilde{d}_{\mathcal{M}}\left(f_{n}(\mathbf{X}), f_{m}(\mathbf{X})\right) d(\text { vol }) \leq \\
& \leq \int_{\mathcal{B}_{0}} d_{\mathcal{M}}\left(f_{n}(\mathbf{X}), f_{m}(\mathbf{X})\right) d(v o l)= \\
& =9\left(\frac{1}{n+1}-\frac{1}{m+1}\right)
\end{aligned}
$$

so that the sequence $f_{n}$ is Cauchy's. However, if we let $n$ to infinity, the resulting limit is

$$
f(\mathbf{X})=\left\{\begin{array}{l}
0 \quad, \quad \text { if }-1<X_{1}<1 \\
1 \quad, \quad \text { if } 1 \leq X_{1}<2
\end{array},\right.
$$

which is not continuous. The physical situation covered by this example is the one of a two-phase material in which the order parameter is the void volume fraction of one of the two species. The limiting distribution (10) describes the complete separation of the two species with the occurrence of a discontinuity surface in the plane $X_{1}=1$. 
(ii) Case 2: $\mathcal{M}$ is compact. Let us assume $\mathcal{M} \equiv S^{1} \subset \mathbb{C}$ and define with a slight abuse of notation

$$
\boldsymbol{\nu}_{n} \equiv f_{n}(\mathbf{X})=\left\{\begin{array}{cc}
0 \quad, \quad \text { if }-1<X_{1} \leq 0 \\
\exp \left(i X_{1}^{n}\right), \quad \text { if } 0 \leq X_{1} \leq 1 \\
\exp (i), \quad \text { if } 1 \leq X_{1}<2
\end{array}\right.
$$

for $\left(X_{2}, X_{3}\right)$ ranging in $(-1,2)^{2}$, with $i$ the imaginary unit, and $\tilde{d}_{\mathcal{M}}(a, b)=$ $d_{\mathcal{M}}(a, b)=|a-b|$. For $n \leq m$, we get

$$
\begin{aligned}
d^{(i)}\left(f_{n}, f_{m}\right) & =\int_{\mathcal{B}_{0}} \tilde{d}_{\mathcal{M}}\left(f_{n}(\mathbf{X}), f_{m}(\mathbf{X})\right) d(v o l)= \\
& =9 \int_{0}^{1} \sqrt{2\left(1-\cos \left(X_{1}^{n}-X_{1}^{m}\right)\right)} d X_{1} \leq \\
& \leq 9 \int_{0}^{1}\left(X_{1}^{n}-X_{1}^{m}\right) d X_{1}=9\left(\frac{1}{n+1}-\frac{1}{m+1}\right)
\end{aligned}
$$

so that the sequence $f_{n}$ is Cauchy's but, as $n \rightarrow+\infty$, its limit $f(\mathbf{X})$, with

$$
f(\mathbf{X})=\left\{\begin{array}{c}
0, \quad \text { if }-1<X_{1}<1 \\
\exp (i) \quad, \quad \text { if } 1 \leq X_{1}<2
\end{array}\right.
$$

is not continuous. The physical situation covered by such an example could be (rather ideally) the one of a porous medium with connected voids full of normal superfluid Helium (i.e., ${ }^{4} \mathrm{He}$; see [4] pag. 7) which, to the limit (13), percolates on one side with respect to the plane $X_{1}=1$.

Remark 5. The metric (5) may furnish unreasonable results for unbounded bodies. In fact, let us consider a Cartesian frame $0 X_{1} X_{2} X_{3}$, and a domain $B=D_{q} \times[0,+\infty)$ where $D_{q}$ is the square $(-1,1)^{2}$ in the plane $X_{3}=0$. Imagine that the closure of the domain $B$ is occupied by a semiinfinite beam made of a two-phase material so that the order parameter is a scalar ranging in $[0,1]$ and representing the volume fraction of one phase. We consider a distribution of the two phases homogeneous with respect to $X_{3}$ so that in a given configuration the distribution of the phases is expressed by the order parameter mapping $B \ni\left(X_{1}, X_{2}, X_{3}\right) \rightarrow \nu_{1}=\tilde{\nu}_{1}\left(X_{1}, X_{2}\right) \in$ $\mathcal{M} \equiv[0,1]$. We consider another distribution of the two phases, namely $\tilde{\nu}_{2}=1-\tilde{\nu}_{1}$ and select $\tilde{d}_{\mathcal{M}}(x, y)=d_{\mathcal{M}}(x, y)=|x-y|$ so that the integral $\int_{D_{q}} d_{\mathcal{M}}\left(\nu_{1}, \nu_{2}\right) d($ area $)=\int_{D_{q}}\left|2 \nu_{1}-1\right| d($ area $)$ is a certain constant positive $K$ (when $\nu_{1}$ is not a constant equal to $\left.\frac{1}{2}\right)$, then the distance $d^{(i)}\left(\tilde{\nu}_{1}, \tilde{\nu}_{2}\right)$ reduces to the integral of $K$ over $[0,+\infty)$ which is $+\infty$. In other words, the two configurations obtained through a rigid rotation of the beam are at infinite distance if one uses the integral distance (5). The same result does not occur with the distances (6) and (7) and the calculation reduces basically to the evaluation of the maximum of the function $\left|2 \tilde{\nu}_{1}-1\right|$ varying $\left(X_{1}, X_{2}\right)$ in $D_{q}$. 
To control the behavior of the derivatives of $\boldsymbol{\nu}$, basically of $\nabla \tilde{\boldsymbol{\nu}}$ with $\nabla \boldsymbol{\nu} \equiv \nabla \tilde{\boldsymbol{\nu}}(\mathbf{X}) \in \operatorname{Hom}\left(T_{\mathbf{X}} \mathcal{B}_{0}, T_{\boldsymbol{\nu}} \mathcal{M}\right)$, we find problematic to act directly on $\operatorname{Hom}\left(T \mathcal{B}_{0}, T \mathcal{M}\right)$. In fact, when we select $\tilde{\boldsymbol{\nu}}_{1}$ and $\tilde{\boldsymbol{\nu}}_{2}$, at a given $\mathbf{X}$, we get in general $\boldsymbol{\nu}_{1} \neq \boldsymbol{\nu}_{2}$ so that we get $\nabla \boldsymbol{\nu}_{1} \in \operatorname{Hom}\left(T_{\mathbf{X}} \mathcal{B}_{0}, T_{\boldsymbol{\nu}_{1}} \mathcal{M}\right)$ and $\nabla \boldsymbol{\nu}_{2} \in \operatorname{Hom}\left(T_{\mathbf{X}} \mathcal{B}_{0}, T_{\boldsymbol{\nu}_{2}} \mathcal{M}\right)$. Though $\mathbf{g}_{\mathcal{M}}$ induces natural metric structures over $T^{*} \mathcal{M}$ and tensor product bundles of $T \mathcal{M}$ and $T^{*} \mathcal{M}$, it does not give a natural way of comparing $\nabla \boldsymbol{\nu}_{1}$ and $\nabla \boldsymbol{\nu}_{2}$ because $\nabla \boldsymbol{\nu}$ is in certain sense like the two-point tensor $\mathbf{F}$. To compare them it is then necessary to transport $\nabla \boldsymbol{\nu}_{2}$ over $\boldsymbol{\nu}_{1}$ through a connection or $\nabla \boldsymbol{\nu}_{1}$ over $\boldsymbol{\nu}_{2}$, i.e. to transfer elements of $T_{\boldsymbol{\nu}_{2}} \mathcal{M}$ over $T_{\boldsymbol{\nu}_{1}} \mathcal{M}$. Since we do not assign any prevalent rôle to Levi-Civita connection, even in the case in which $\mathcal{M}$ is complete and we may connect $\boldsymbol{\nu}_{1}$ and $\boldsymbol{\nu}_{2}$ with a geodetic, we face the circumstance that the transport is in general not isometric, so that the comparison (defined in some way) of the two derivatives at $\boldsymbol{\nu}_{1}$ may lead to a different result of the same comparison at $\boldsymbol{\nu}_{2}$. Moreover, even in the case in which one would choose to use both the comparison at $\boldsymbol{\nu}_{1}$ and the one at $\boldsymbol{\nu}_{2}$, say, using half of their sum, one could face the basic difficulty that the transport could be unbounded (due, e.g., to topological features of $\mathcal{M}$ ) or the bound could not be uniform over the curve connecting $\boldsymbol{\nu}_{1}$ with $\boldsymbol{\nu}_{2}$. In this case, the possible choice to consider admissible only values over $\mathcal{M}$ that can be connected by curves assuring a uniformly bounded transport could reduce too much the generality of $\mathcal{M}$.

By considering previous remarks, we follow a procedure leading to the possibility to compare $\nabla \boldsymbol{\nu}_{1}$ and $\nabla \boldsymbol{\nu}_{2}$ in $\operatorname{Hom}\left(T_{\mathbf{X}} \mathcal{B}_{0}, T_{\mathbf{X}}^{*} \mathcal{B}_{0}\right)$.

To this aim, we consider first the adjoint of $\nabla \tilde{\boldsymbol{\nu}}$, indicated with $\nabla \tilde{\boldsymbol{\nu}}^{*}$ and such that $\nabla \boldsymbol{\nu}^{*} \equiv \nabla \tilde{\boldsymbol{\nu}}^{*}(\mathbf{X}) \in \operatorname{Hom}\left(T_{\boldsymbol{\nu}_{1}}^{*} \mathcal{M}, T_{\mathbf{X}}^{*} \mathcal{B}_{0}\right)$, then we define

$$
\bar{d}_{\mathcal{M}}\left(\nabla \boldsymbol{\nu}_{1}, \nabla \boldsymbol{\nu}_{2}\right)=\left\|\nabla \boldsymbol{\nu}_{1}^{*} \nabla \boldsymbol{\nu}_{1}-\nabla \boldsymbol{\nu}_{2}^{*} \nabla \boldsymbol{\nu}_{2}\right\|
$$

where here $\|\cdot\|$ is the usual norm in $\mathbb{R}^{3} \otimes \mathbb{R}^{3}$, i.e. the usual norm of $3 \times 3$ matrices such that, for any $\mathbf{A} \in \mathbb{R}^{3} \otimes \mathbb{R}^{3}$, one has $\|\mathbf{A}\|=\sqrt{\operatorname{tr}\left(\mathbf{A}^{T} \mathbf{A}\right)}$.

Remark 6. We express $\nabla \tilde{\boldsymbol{\nu}}^{*} \nabla \tilde{\boldsymbol{\nu}}$ as $\left(\nabla \tilde{\boldsymbol{\nu}}^{*} \nabla \tilde{\boldsymbol{\nu}}\right)_{A B}=\nabla \tilde{\boldsymbol{\nu}}_{A}^{* \alpha}\left(\mathbf{g}_{\mathcal{M}}\right)_{\alpha \beta} \nabla \tilde{\boldsymbol{\nu}}_{B}^{\beta}$ in components. In other words, the second order tensor $\nabla \tilde{\boldsymbol{\nu}}^{*} \nabla \tilde{\boldsymbol{\nu}}$ is the pull-back in $\mathcal{B}_{0}$ of the metric $\mathbf{g}_{\mathcal{M}}$ in $\mathcal{M}$. As a consequence, the distance $\bar{d}_{\mathcal{M}}\left(\nabla \boldsymbol{\nu}_{1}, \nabla \boldsymbol{\nu}_{2}\right)$ compares values of the metric $\mathbf{g}_{\mathcal{M}}$ at two different points (namely $\boldsymbol{\nu}_{1}$ and $\boldsymbol{\nu}_{2}$ ) of $\mathcal{M}$. In fact, the scalar product $\nabla \boldsymbol{\nu}_{1}^{*} \nabla \boldsymbol{\nu}_{1} \cdot(d \mathbf{X} \otimes d \mathbf{X})$ is the 'length' of $d \boldsymbol{\nu}$ at $\boldsymbol{\nu}_{1}$.

With $\bar{d}_{\mathcal{M}}$ as above, we define the counterparts of the distances (5), (6) and (7).

4. The first distance, indicated with $\bar{d}^{(i)}$, is defined by

$$
\bar{d}^{(i)}\left(\nabla \tilde{\boldsymbol{\nu}}_{1}, \nabla \tilde{\boldsymbol{\nu}}_{2}\right)=\int_{\mathcal{B}_{0}} \bar{d}_{\mathcal{M}}\left(\nabla \boldsymbol{\nu}_{1}, \nabla \boldsymbol{\nu}_{2}\right) d(\text { vol }) .
$$

5. Let $\left\{K_{n}\right\}$ be an exhaustion of $\mathcal{B}_{0}$, i.e. a compact cover of $\mathcal{B}_{0}$ such that $K_{n} \subset \stackrel{\circ}{K}_{n+1}$ for any $n$. The second distance, indicated with $\bar{d}^{(c)}$, is 
defined by

$$
\bar{d}^{(c)}\left(\nabla \tilde{\boldsymbol{\nu}}_{1}, \nabla \tilde{\boldsymbol{\nu}}_{2}\right)=\sum_{n \in \mathbb{N}} \frac{1}{2^{-n}} \max _{\mathbf{X} \in K_{n}} \bar{d}_{\mathcal{M}}\left(\nabla \boldsymbol{\nu}_{1}, \nabla \boldsymbol{\nu}_{2}\right),
$$

6. More simply, a third natural distance, indicated with $d^{(s)}$, can be defined by

$$
\bar{d}^{(s)}\left(\nabla \tilde{\boldsymbol{\nu}}_{1}, \nabla \tilde{\boldsymbol{\nu}}_{2}\right)=\sup _{\mathbf{X} \in \mathcal{B}_{0}} \bar{d}_{\mathcal{M}}\left(\nabla \boldsymbol{\nu}_{1}, \nabla \boldsymbol{\nu}_{2}\right),
$$

when $\sup _{\mathbf{X} \in \mathcal{B}_{0}} \bar{d}_{\mathcal{M}}$ remains bounded over $\mathcal{B}_{0}$.

Remark 7. We do not introduce any 'normalization' of the distance like the one in (44). In fact, (15)-(17) are calculated over bundles whose fibers are linear spaces so that $\bar{d}_{\mathcal{M}}(\cdot, \cdot)$ displays possible properties of homogeneity (natural over fiber spaces) while a normalization like (4) would not.

Appropriate topologies on $\mathcal{C}_{\boldsymbol{\nu}}$ may then be induced by the distances

$$
\begin{aligned}
& \tilde{d}^{(i)}\left(\tilde{\boldsymbol{\nu}}_{1}, \tilde{\boldsymbol{\nu}}_{2}\right)=d^{(i)}\left(\tilde{\boldsymbol{\nu}}_{1}, \tilde{\boldsymbol{\nu}}_{2}\right)+\bar{d}^{(i)}\left(\nabla \tilde{\boldsymbol{\nu}}_{1}, \nabla \tilde{\boldsymbol{\nu}}_{2}\right), \\
& \tilde{d}^{(c)}\left(\tilde{\boldsymbol{\nu}}_{1}, \tilde{\boldsymbol{\nu}}_{2}\right)=d^{(c)}\left(\tilde{\boldsymbol{\nu}}_{1}, \tilde{\boldsymbol{\nu}}_{2}\right)+\bar{d}^{(c)}\left(\nabla \tilde{\boldsymbol{\nu}}_{1}, \nabla \tilde{\boldsymbol{\nu}}_{2}\right), \\
& \tilde{d}^{(s)}\left(\tilde{\boldsymbol{\nu}}_{1}, \tilde{\boldsymbol{\nu}}_{2}\right)=d^{(s)}\left(\tilde{\boldsymbol{\nu}}_{1}, \tilde{\boldsymbol{\nu}}_{2}\right)+\bar{d}^{(s)}\left(\nabla \tilde{\boldsymbol{\nu}}_{1}, \nabla \tilde{\boldsymbol{\nu}}_{2}\right) .
\end{aligned}
$$

Remark 8. A program based on the systematic analysis of the properties of $\mathcal{C}_{\boldsymbol{\nu}}$ associated with each metric above could deserve to be followed. In fact one could analyze systematically the influence of the topological properties of $\mathcal{M}$ (such as compactness) on the structure of $\mathcal{C}_{\boldsymbol{\nu}}$. Here, such a program goes far from the main purposes of the present paper and we do not follow it. However, the remarks above underline the nature of subtle geometrical difficulties that one may encounter in going on and constitute a reasonable starting point for further investigations. In any case, one should take into account that when the manifold $\mathcal{M}$ is per se a linear space or for reasons of modeling is embedded isometrically in a linear space, the results in [2] and [18] apply directly to characterize the topological properties of the space $\mathcal{C}$.

\subsection{Something more about kinematics}

Motions are sufficiently smooth curves over $\mathcal{C}$. For a given interval of time $[0, \bar{t}]$, we then have mappings $[0, \bar{t}] \ni t \longmapsto\left(\tilde{\mathbf{x}}_{t}, \tilde{\boldsymbol{\nu}}_{t}\right) \in \mathcal{C}$ and indicate with $\mathbf{x}=\tilde{\mathbf{x}}(\mathbf{X}, t)$ and $\boldsymbol{\nu}=\tilde{\boldsymbol{\nu}}(\mathbf{X}, t)$ the current place at $t$ of a material element resting at $\mathbf{X}$ when $t=0$ and the current value of the order parameter.

With $\dot{\mathbf{X}}$ and $\dot{\boldsymbol{\nu}}$ we denote rates given by $\frac{d \tilde{\mathbf{x}}}{d t}(\mathbf{X}, t)$ and $\frac{d \tilde{\boldsymbol{\nu}}}{d t}(\mathbf{X}, t)$ respectively, with $\dot{\boldsymbol{\nu}} \in T_{\tilde{\boldsymbol{\nu}}(\mathbf{X}, t)} \mathcal{M}$. They have counterparts $\mathbf{v}$ and $\boldsymbol{v}$ in the current place $\mathcal{B}$ given by

$$
\mathcal{B} \times[0, \bar{t}] \ni(\mathbf{x}, t) \stackrel{\tilde{\mathbf{v}}}{\longmapsto} \mathbf{v}=\tilde{\mathbf{v}}(\mathbf{x}, t) \in T_{\mathbf{x}} \mathcal{B}
$$


and

$$
\mathcal{B} \times[0, \bar{t}] \ni(\mathbf{x}, t) \stackrel{\tilde{\boldsymbol{v}}}{\longmapsto} \boldsymbol{v}=\tilde{\boldsymbol{v}}(\mathbf{x}, t) \in T_{\boldsymbol{\nu}(\mathbf{x}(\mathbf{X}, t), t)} \mathcal{M},
$$

obtained through the mapping $\mathbf{X} \longmapsto \mathbf{x}=\tilde{\mathbf{x}}(\mathbf{X})$ at each $t$. We have $\dot{\mathbf{x}}=\mathbf{v}$ but $\boldsymbol{v}=\dot{\boldsymbol{\nu}}+(\operatorname{grad} \boldsymbol{\nu}) \mathbf{v}$ and we may write also $\boldsymbol{v}=\dot{\boldsymbol{\nu}}+(\nabla \boldsymbol{\nu}) \mathbf{F}^{-1} \mathbf{v}=\dot{\boldsymbol{\nu}}-$ $(\nabla \boldsymbol{\nu}) \dot{\mathbf{X}}$, where $\dot{\mathbf{X}}$ is the material velocity $-\mathbf{F}^{-1} \mathbf{v}$ associated with the inverse mapping $\mathbf{X}=\tilde{\mathbf{x}}^{-1}(\mathbf{x}, t)$.

For the acceleration of the order parameter we have

$$
\ddot{\nu}^{\alpha}=\partial_{t} \dot{\nu}^{\alpha}+\breve{\Gamma}_{\beta \gamma}^{\alpha} \dot{\nu}^{\beta} \dot{\nu}^{\gamma}
$$

with

$$
\breve{\Gamma}_{\beta \gamma}^{\alpha}=\frac{1}{2} \mathbf{g}_{\mathcal{M}}^{\alpha \delta}\left(\partial_{\nu^{\gamma}}\left(\mathbf{g}_{\mathcal{M}}\right)_{\beta \delta}+\partial_{\nu^{\beta}}\left(\mathbf{g}_{\mathcal{M}}\right)_{\gamma \delta}-\partial_{\nu^{\delta}}\left(\mathbf{g}_{\mathcal{M}}\right)_{\beta \gamma}\right)
$$

Christoffel symbols relevant for $\mathcal{M}$. The expression (23) enters the representation of possible inertial terms pertaining to the substructure ${ }^{2}$.

Let $\mathbf{g}$ be the metric in the ambient space (in general, for curved frames we have $\mathbf{x} \longmapsto \mathbf{g}(\mathbf{x})$, i.e. $\mathbf{g}$ depends on the place), by indicating with $\mathbf{F}^{T}$ the transpose of $\mathbf{F}$, the mapping

$$
\mathcal{B}_{0} \ni \mathbf{X} \longmapsto\left(\mathbf{F}^{T} \mathbf{F}\right) \equiv \mathbf{C}(\mathbf{X}) \in \operatorname{Sym}^{+}\left(T_{\mathbf{X}} \mathcal{B}_{0}, T_{\mathbf{X}}^{*} \mathcal{B}_{0}\right)
$$

is the pull-back of $\mathbf{g}$ through the deformation $\tilde{\mathbf{x}}$ and in coordinates we have $C_{A B}=\left(F^{T}\right)_{A}{ }^{i} g_{i j} F_{B}^{j}$.

If $\mathcal{B}_{0}$ is endowed with a not flat 'material' metric $\gamma$ (i.e. $\mathcal{B}_{0} \ni \mathbf{X} \stackrel{\tilde{\gamma}}{\longmapsto}$ $\left.\gamma=\tilde{\gamma}(\mathbf{X}) \in \operatorname{Sym}^{+}(V e c, V e c)\right)$, the difference $(\mathbf{C}-\boldsymbol{\gamma})(\mathbf{X})$ is twice the nonlinear deformation tensor $\mathbf{E}(\mathbf{X})$ which measures relative changes of lengths by using $\mathcal{B}_{0}$ as paragon setting. In an alternative point of view, we may consider $\mathcal{B}$ as paragon setting pushing forward $\gamma$ and comparing lengths there as explained in all treatises on non-linear elasticity of simple bodies in chapters dealing with measures of deformation.

In the case of complex bodies the matter may be more intricate and a general (in certain sense abstract) treatment of measures of deformation seems to be absent (the only exception being the basic remarks in [5]). The key point is the specific nature of $\boldsymbol{\nu}$. In fact, when $\boldsymbol{\nu}$ represents, e.g., a microdisplacement, an independent rotation or an independent deformation, its gradient enters the measures of deformation together with $\boldsymbol{\nu}$ itself. On the contrary, when $\boldsymbol{\nu}$ describes a property not related strictly with changes of lengths (say in the case in which $\boldsymbol{\nu}$ represents the volume fraction of a phase in a two-phase material or the spontaneous polarization in ferroelectrics etc.), standard $\mathbf{C}$ and $\mathbf{E}$, or their spatial counterparts, are sufficient to measure the macroscopic deformation.

In general, we could imagine to have a map of the type

$$
(\mathbf{F}, \mathbf{g}, \boldsymbol{\nu}, \nabla \boldsymbol{\nu}) \longmapsto \mathbf{G}(\mathbf{F}, \mathbf{g}, \boldsymbol{\nu}, \nabla \boldsymbol{\nu}) \in S y m^{+}\left(T_{\mathbf{X}} \mathcal{B}_{0}, T_{\mathbf{X}}^{*} \mathcal{B}_{0}\right)
$$

\footnotetext{
${ }^{2}$ Basic remarks about the influence of $\breve{\boldsymbol{\Gamma}}$ on inertia are discussed in [7].
} 
with $\mathbf{G}$ a metric in $\mathcal{B}_{0}$ involving the pull-back of $\mathbf{g}$, and to define a deformation tensor $\overline{\mathbf{E}}(\mathbf{X})$ as $\frac{1}{2}(\mathbf{G}-\boldsymbol{\gamma})(\mathbf{X})$, erasing $\boldsymbol{\nu}$ and/or $\nabla \boldsymbol{\nu}$ each time in which circumstances suggest such a cancellation.

Of course, the information furnished by (26) is rather volatile unless we put it in a context specifying its nature for some particular substructure.

Remark 9. Cosserat [10] and micromorphic ([28]) materials are well known classes of complex bodies in which the order parameter (or its gradient) affects the representation of the measures of deformation (see also essential remarks in [4]). In the former class each material element is pictured as a 'small' rigid body that may undergo rotations independent of the surrounding material elements. Of course, neighboring relative rotations may alter lengths, in a sense described e.g. in [10] or [4]. In the latter class one imagines that each material element may suffer independent deformations (it is like a ball of rubber). Measures of relative deformations may be then introduced. Less popular is the case of microcracked bodies and we give some details about it in order to construct an explicit example of the possible influence of the order parameter and its gradient on the measures of deformation. In fact, when microcracks are smeared throughout a body, the material element is pictured as a 'patch' of matter endowed with a population of microcracks that can be considered either as planar sharp defects not interpenetrated by interatomic bonds or as elliptic voids with non-null volume and one dimension very small with respect to the others. If we consider frozen the microcracks in a given material element placed at $\mathbf{X}$ in $\mathcal{B}_{0}$, a standard deformation $\tilde{\mathbf{x}}$ puts it (or better its centre of mass) in the place $\mathbf{x}=\tilde{\mathbf{x}}(\mathbf{X})$. Now, if we allow the microcracks to deform (say without growing irreversibly for the sake of simplicity), the centre of mass of each material element undergoes in principle a shift ${ }^{3}$ toward a new place $\mathbf{x}^{\prime}$. If we indicate with $\mathcal{B}^{\prime}$ the minimal regular region containing the collection of $\mathbf{x}^{\prime}$, each one corresponding to each $\mathbf{x}$, we may imagine (this is a basic point of modeling) to obtain $\mathcal{B}^{\prime}$ from $\mathcal{B}$ by means of a sufficiently smooth mapping $\mathfrak{f}$ such that $\mathbf{x}^{\prime}=(\mathfrak{f} \circ \tilde{\mathbf{x}})(\mathbf{X})$ and $\mathfrak{f}(\mathcal{B})=\mathcal{B}^{\prime}$. By denoting with grad the gradient with respect to $\mathbf{x}$, as before, by chain rule we get $\nabla(\mathfrak{f} \circ \tilde{\mathbf{x}})(\mathbf{X})=((\operatorname{grad} \mathfrak{f}) \nabla \tilde{\mathbf{x}})(\mathbf{X})=\mathbf{F}^{(m)} \mathbf{F}$, where $\mathbf{F}^{(m)}=(\operatorname{grad} \mathfrak{f})(\mathbf{x})$ is the gradient of deformation from $\mathcal{B}$ to $\mathcal{B}^{\prime}$, i.e., $\mathbf{F}^{(m)}=\operatorname{Hom}\left(T_{\mathbf{x}} \mathcal{B}, T_{\mathbf{x}^{\prime}} \mathcal{B}^{\prime}\right)$. If we indicate with $\mathbf{d}(\mathbf{X})=(\mathfrak{f} \circ \tilde{\mathbf{x}})(\mathbf{X})-\tilde{\mathbf{x}}(\mathbf{X})$ the displacement from $\mathbf{x}$ to $\mathbf{x}^{\prime}$, defined as a field over $\mathcal{B}_{0}$, since $\nabla \mathrm{d}(\mathbf{X})=\left(\operatorname{gradd}_{a}\right) \mathbf{F}$, with $\mathrm{d}_{a}=\mathrm{d} \circ \tilde{\mathbf{x}}^{-1}$, we get the additive decomposition $\nabla(\mathfrak{f} \circ \tilde{\mathbf{x}})(\mathbf{X})=\mathbf{F}+\nabla \mathrm{d}(\mathbf{X})$. If we write $\mathbf{F}_{t o t}=\mathbf{F}+\nabla \mathrm{d}(\mathbf{X}), \mathbf{F}_{t o t}$ is the gradient of deformation from $\mathcal{B}_{0}$ to $\mathcal{B}^{\prime}$. So that the right Cauchy-Green tensor $\mathbf{C}_{t o t}(\mathbf{X})=\mathbf{F}_{\text {tot }}^{T} \mathbf{F}_{\text {tot }}$ involves $\nabla \mathrm{d}$. Moreover, by comparing the additive decomposition of $\mathbf{F}_{t o t}$ with the multiplicative one, namely $\mathbf{F}^{(m)} \mathbf{F}$, we realize that $\mathbf{F}^{(m)}=\mathbf{I}+\nabla \mathrm{d}(\mathbf{X}) \mathbf{F}^{-1}=\mathbf{I}+\operatorname{gradd}_{a}$. The direct (perhaps naive) description of the kinematics of microcracked

\footnotetext{
${ }^{3}$ Here the circumstance that there is a population of microcracks in the material element and that each microcrack is not a spheric void is crucial.
} 
bodies just sketched here follows [21], [26]; however, it can be obtained by using the procedure involving the limit of bodies described in [12], [13].

\subsection{Observers}

Three geometrical environments are necessary to describe a motion and then the configuration at a prescribed instant of a complex body: the time interval $[0, \bar{t}]$ (that we could extend in principle to the whole real line), the ambient space $\mathcal{E}^{3}$ (of course $\mathcal{E}^{2}$ or $\mathcal{E}^{1}$ if we deal with two- or one-dimensional bodies), and the manifold $\mathcal{M}$.

In classical mechanics an observer is a representation of the measure of time and the ambient space so that changes of observers are changes of representation.

Here, our point of view is that the notion of observer should involve all the descriptors of the morphology (i.e. the geometry) of the material element. Then, for us, an observer $\mathcal{O}$ is a representation of a triplet of geometrical settings: (i) the interval of time, (ii) the ambient space $\mathcal{E}^{3}$ and (iii) the manifold $\mathcal{M}$ of substructural states [25]. Such a definition is a natural extension of the standard point of view including just (i) and (ii): it takes into account the presence of the manifold of substructural states $\mathcal{M}$ as natural geometric ingredient in the representation of the body.

For the sake of simplicity we shall consider observers coinciding about the measure of time.

General changes of observers, as used below, will then involve automorphism of $\mathcal{E}^{3}$ and the action of arbitrary Lie groups over $\mathcal{M}$. In this sense the notion of change of observers involves a pair of one-parameter families of transformations indicated below.

A1. $\mathbb{R}^{+} \ni s_{2} \longmapsto \mathbf{f}_{s_{2}}^{2} \in \operatorname{Aut}\left(\mathcal{E}^{3}\right)$, with $\mathbf{f}_{0}^{2}$ the identity ${ }^{4}$. We put $\mathbf{f}_{0}^{2 \prime}(\mathbf{X})=\mathbf{v}$, where the prime denotes differentiation with respect to $s_{2}$.

A2. A Lie group $G$, with Lie algebra $\mathfrak{g}$, acts over $\mathcal{M}$. If $\xi \in \mathfrak{g}$, its action over $\boldsymbol{\nu} \in \mathcal{M}$ is indicated with $\xi_{\mathcal{M}}(\boldsymbol{\nu})$. By indicating with $\boldsymbol{\nu}_{g}$ the value of $\boldsymbol{\nu}$ after the action of $g \in G$, if we consider a one-parameter smooth curve $\mathbb{R}^{+} \ni s_{3} \longmapsto g_{s_{3}} \in G$ over $G$, such that $\xi=\left.\frac{d g_{s_{3}}}{d s_{3}}\right|_{s_{3}=0}$, and its corresponding curve $s_{3} \longmapsto \boldsymbol{\nu}_{g_{s_{3}}}$ over $\mathcal{M}$, starting from a given $\boldsymbol{\nu}$, we have $\xi_{\mathcal{M}}(\boldsymbol{\nu})=\left.\frac{d}{d s_{3}} \boldsymbol{\nu}_{g_{s_{3}}}\right|_{s_{3}=0}$. When $G$ coincides with $S O$ (3), then $\xi_{\mathcal{M}}(\boldsymbol{\nu}) \equiv \mathcal{A}(\boldsymbol{\nu}) \dot{\mathbf{q}}$, with $\mathcal{A}(\boldsymbol{\nu})$ defined above and $\dot{\mathbf{q}} \wedge \in \mathfrak{s o}(3)$.

We specify for future use in special cases the relations characterizing 'rigid' changes of observers, i.e. the ones characterized by the contemporary action of $S O(3)$ on $\mathcal{M}$ and $\mathcal{E}^{3}$. The latter action is induced by standard isometries of point space.

Let $[0, \bar{t}] \ni t \longmapsto \mathbf{Q}(t) \in S O(3)$, with $\mathbf{Q}(0)=\operatorname{Id}_{S O(3)}$, be a smooth curve on $S O(3)$. Let also $\mathcal{O}$ be an assigned representation of $\mathcal{E}^{3}$ at $t=0$.

\footnotetext{
${ }^{4}$ Aut $\left(\mathcal{E}^{3}\right)$ is the group of automorphysms of $\mathcal{E}^{3}$.
} 
At any $t \neq 0$ we may associate an observer $\mathcal{O}_{t}^{\prime}$ obtained from $\mathcal{O}$ by means of $\mathbf{Q}(t)$. The transformation from $\mathcal{O}$ to $\mathcal{O}_{t}^{\prime}$ is isometric, then a point $\mathbf{x}$ seen by $\mathcal{O}$ is mapped in a point $\mathbf{x}^{\prime}=\mathbf{w}(t)+\mathbf{Q}(t)\left(\mathbf{x}-\mathbf{x}_{0}\right)$, where $t \longmapsto \mathbf{w}(t)$ is an arbitrary point valued function smooth in time and $\mathbf{x}_{0}$ a fixed point chosen at will in space. If we calculate the rate of $\mathbf{x}^{\prime}$ and pull-back it in $\mathcal{O}$ writing $\dot{\mathbf{x}}^{*}$ for $\mathbf{Q}^{T} \dot{\mathbf{x}}^{\prime}$, we get the standard reaction

$$
\dot{\mathbf{x}}^{*}=\dot{\mathbf{x}}+\mathbf{c}(t)+\dot{\mathbf{q}} \wedge\left(\mathbf{x}-\mathbf{x}_{0}\right),
$$

characterizing a classical change of observer, where $\mathbf{c}=\mathbf{Q}^{T} \mathbf{w}$ is the translational velocity and $\dot{\mathbf{q}}$ the rotational one. If the arbitrary element $\dot{\mathbf{q}} \wedge$ of the Lie algebra $\mathfrak{s o}(3)$ acts also on $\mathcal{M}$, it induces a rate (that we call 'rigid', with the subscript $R$, just for reminding the circumstance that it is related to a spatial rigid body motion) given by $\mathcal{A} \dot{\mathbf{q}}$, where $\mathcal{A}(\boldsymbol{\nu}) \in \operatorname{Hom}\left(V e c, T_{\boldsymbol{\nu}} \mathcal{M}\right)$. Classical changes of observer in a multifield setting are then characterized by the relations (27) and

$$
\dot{\nu}^{*}=\dot{\nu}+\mathcal{A} \dot{\mathbf{q}}
$$

when they agree about the measure of time. Basically, if $\boldsymbol{\nu}_{q}$ denotes the value of $\boldsymbol{\nu}$ after the right action of $S O(3)$ over $\mathcal{M}$, we have $\mathcal{A}=\left.\frac{d \boldsymbol{\nu}_{q}}{d \mathbf{q}}\right|_{\mathbf{q}=0}$, where $\mathbf{q}$ is a vector connected with $\mathbf{Q} \in S O(3)$ by the formula $\mathbf{Q}=\exp (-\mathbf{e q})$.with e Ricci's permutation index. For example, let $\mathcal{M}$ be coincident with $S^{2}$ as in the case of magnetostrictive materials). For any $\tau \in S^{2}$, we get $\tau_{\mathbf{q}}=\mathbf{Q} \boldsymbol{\tau}$, then $\mathcal{A}=-\boldsymbol{\tau} \wedge$.

\subsection{Relabeling}

Another transformation playing a role in the developments presented in what follows is the relabeling of material elements in the reference place $\mathcal{B}_{0}$. With the term 'relabeling' we indicate a generic transformation of $\mathcal{B}_{0}$ induced by a $C^{1}\left(\mathcal{B}_{0}\right)$ point valued mapping $\mathbf{f}^{1}$ such that (i) it is isocoric (i.e. it preserves volume) and (ii) establishes also a diffeomorphism between $\mathcal{B}_{0}$ and $\mathbf{f}^{1}\left(\mathcal{B}_{0}\right)$. From a physical point of view, the important part of the action of $\mathbf{f}^{1}$ relies in a sort of 'permutation' of possible defects or, better, inhomogeneities in $\mathcal{B}_{0}$. In particular, we will consider a one-parameter family of such isocoric diffeomorphisms defined below:

A3. $\mathbb{R}^{+} \ni s_{1} \longmapsto \mathbf{f}_{s_{1}}^{1} \in \operatorname{SDiff}\left(\mathcal{B}_{0}\right)$, with $\mathbf{f}_{0}^{1}$ the identity; i.e. at each $s_{1}$

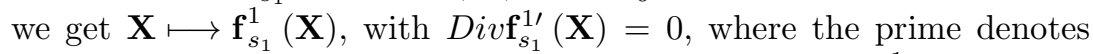
differentiation with respect to the parameter $s_{1}$. We put $\mathbf{f}_{0}^{1 \prime}(\mathbf{X})=\mathbf{w}$.

\section{Langrangian $3+1$ forms and balance equations}

The multifield theoretical analogue of the theory of elasticity for complex bodies (i.e., bodies with material substructure) relies on rather articulated fiber bundles. 
We start by considering a fiber bundle $\pi: \mathcal{Y} \rightarrow \mathcal{B}_{0} \times[0, \bar{t}]$ such that $\pi^{-1}(\mathbf{X}, t)=\mathcal{E}^{3} \times \mathcal{M}$. A generic section $\eta \in \Gamma(\mathcal{Y})$ of $\mathcal{Y}$ is then $\eta(\mathbf{X}, t)=$ $(\mathbf{X}, t, \mathbf{x}, \boldsymbol{\nu})$. For sufficiently smooth sections, the first jet bundle $J^{1} \mathcal{Y}$ over $\mathcal{Y}$ is such that $J^{1} \mathcal{Y} \ni j^{1}(\eta)(\mathbf{X}, t)=(\mathbf{X}, t, \mathbf{x}, \dot{\mathbf{x}}, \mathbf{F}, \boldsymbol{\nu}, \dot{\boldsymbol{\nu}}, \nabla \boldsymbol{\nu})$.

Up to this point the discussion has been purely geometric. No issues related with the constitutive nature of the body and with the interactions arising inside it have been discussed. They enter into play here: we assume that the general body under examination is made of (non-linear) elastic material. In this case we may associate with it the canonical Lagrangian $3+1$ form

$$
L: J^{1} \mathcal{Y} \rightarrow \wedge^{3+1}\left(\mathcal{B}_{0} \times[0, \bar{t}]\right)
$$

The definition of the elements of the space of $3+1$ forms $\wedge^{3+1}\left(\mathcal{B}_{0} \times[0, \bar{t}]\right)$ would require some care. In fact, $\mathcal{B}_{0} \times[0, \bar{t}]$ is a manifold with boundary coinciding only with $\{0\} \times \mathcal{B}_{0} \cup\{\bar{t}\} \times \mathcal{B}_{0}$ (because $\mathcal{B}_{0}$ is open and coincides with the interior of its closure), while the definition of odd forms is immediate on manifolds without boundary. However, one is commonly interested in evaluating the variation of the total Lagrangian

$$
\bar{L}\left(\mathcal{B}_{0}\right)=\int_{\mathcal{B}_{0} \times[0, \bar{t}]} \mathcal{L} d^{3} \mathbf{X} \wedge d t
$$

so that, in defining $L\left(j^{1}(\eta)\right)$, possible problems related with boundary points do not play any rôle.

We assume that $L$ admits a density $\mathcal{L}$ such that

$$
L\left(j^{1}(\eta)(\mathbf{X}, t)\right)=\mathcal{L}(\mathbf{X}, \mathbf{x}, \dot{\mathbf{x}}, \mathbf{F}, \boldsymbol{\nu}, \dot{\boldsymbol{\nu}}, \nabla \boldsymbol{\nu}) d^{3} \mathbf{X} \wedge d t
$$

with

$\mathcal{L}(\mathbf{X}, \mathbf{x}, \dot{\mathbf{x}}, \mathbf{F}, \boldsymbol{\nu}, \dot{\boldsymbol{\nu}}, \nabla \boldsymbol{\nu})=\frac{1}{2} \rho_{0}|\dot{\mathbf{x}}|^{2}+\rho_{0} \chi(\boldsymbol{\nu}, \dot{\boldsymbol{\nu}})-\rho_{0} e(\mathbf{X}, \mathbf{F}, \boldsymbol{\nu}, \nabla \boldsymbol{\nu})-\rho_{0} w(\mathbf{x}, \boldsymbol{\nu})$,

where $\rho_{0}$ is the referential mass density (conserved during the motion), $\chi$ the substructural kinetic co-energy (needed when physical circumstances prescribe substructural inertia), $e$ the elastic energy density and $w$ the density of the potential of external actions, all per unit mass. The presence of $\boldsymbol{\nu}$ in the list of entries of $\chi$ and $e$ is due to the non-trivial structure of $\mathcal{M}$. In general the elements of $T \mathcal{M}$ cannot be separated invariantly unless a parallelism can be found over $\mathcal{M}$. From classical non-linear field theories we know that $\mathbf{x}$ disappears from the list of entries of $e$ due to reasons of invariance, but $\boldsymbol{\nu}$ does not (see [4]).

We assume that $\mathcal{L}(\cdot)$ be sufficiently smooth so that we may find at least one section $\eta$ satisfying Euler-Lagrange equations

$$
\begin{gathered}
\overline{\partial_{\dot{\mathbf{x}} \mathcal{L}}}=\partial_{\mathbf{x}} \mathcal{L}-D i v \partial_{\mathbf{F}} \mathcal{L}, \\
\dot{\partial_{\dot{\boldsymbol{\nu}} \mathcal{L}}}=\partial_{\boldsymbol{\nu}} \mathcal{L}-D i v \partial_{\nabla \boldsymbol{\nu}} \mathcal{L} .
\end{gathered}
$$


With respect to the families of transformations characterizing relabeling and changes of observers, for the sake of brevity, we indicate with $\mathbf{f}^{1}, \mathbf{f}^{2}$ and $\boldsymbol{\nu}_{g}$ the values $\mathbf{f}_{s_{1}}^{1}(\mathbf{X}), \mathbf{f}_{s_{2}}^{2}(\mathbf{x}), \boldsymbol{\nu}_{g_{s_{3}}}(\mathbf{X})$.

Definition 1 A Lagrangian density $\mathcal{L}$ is invariant with respect to the action of $\mathbf{f}_{s_{1}}^{1}, \mathbf{f}_{s_{2}}^{2}$ and $G$ if

$$
\mathcal{L}(\mathbf{X}, \mathbf{x}, \dot{\mathbf{x}}, \mathbf{F}, \boldsymbol{\nu}, \dot{\boldsymbol{\nu}}, \nabla \boldsymbol{\nu})=
$$$$
=\mathcal{L}\left(\mathbf{f}^{1}, \mathbf{f}^{2},\left(\operatorname{grad} \mathbf{f}^{2}\right) \dot{\mathbf{x}},\left(\operatorname{grad} \mathbf{f}^{2}\right) \mathbf{F}\left(\nabla \mathbf{f}^{1}\right)^{-1}, \boldsymbol{\nu}_{g}, \dot{\boldsymbol{\nu}}_{g},\left(\nabla \boldsymbol{\nu}_{g}\right)\left(\nabla \mathbf{f}^{1}\right)^{-1}\right),
$$

for any $g \in G$ and $s_{1}, s_{2} \in \mathbb{R}^{+}$.

Let $\mathcal{Q}$ and $\mathfrak{F}$ be scalar and vector densities given respectively by

$$
\begin{gathered}
\mathcal{Q}=\partial_{\dot{\mathbf{x}}} \mathcal{L} \cdot(\mathbf{v}-\mathbf{F} \mathbf{w})+\partial_{\dot{\boldsymbol{\nu}}} \mathcal{L} \cdot\left(\xi_{\mathcal{M}}(\boldsymbol{\nu})-(\nabla \boldsymbol{\nu}) \mathbf{w}\right) \\
\mathfrak{F}=\mathcal{L} \mathbf{w}+\left(\partial_{\mathbf{F}} \mathcal{L}\right)^{T}(\mathbf{v}-\mathbf{F} \mathbf{w})+\left(\partial_{\nabla \boldsymbol{\nu}} \mathcal{L}\right)^{T}\left(\xi_{\mathcal{M}}(\boldsymbol{\nu})-(\nabla \boldsymbol{\nu}) \mathbf{w}\right) .
\end{gathered}
$$

Theorem 1 [9]. If the Lagrangian density $\mathcal{L}$ is invariant under $\mathbf{f}_{s_{1}}^{1}, \mathbf{f}_{s_{2}}^{2}$ and $G$, for any $g \in G$ and $s_{1}, s_{2} \in \mathbb{R}^{+}$, then

$$
\dot{\mathcal{Q}}+\operatorname{Div\mathfrak {F}}=0 .
$$

The detailed proof is contained in [9]. Here we remind only that it is based on a direct explicit calculation of the terms in (38) on the basis of (36) and (37), and on the exploitation of the relations

$$
\begin{aligned}
& \left.\frac{d}{d s_{1}} \mathcal{L}\right|_{s_{1}=0, s_{2}=0, s_{3}=0}=0,\left.\quad \frac{d}{d s_{2}} \mathcal{L}\right|_{s_{1}=0, s_{2}=0, s_{3}=0}=0, \\
& \left.\frac{d}{d s_{3}} \mathcal{L}\right|_{s_{1}=0, s_{2}=0, s_{3}=0}=0
\end{aligned}
$$

which are consequences of the request of invariance for $\mathcal{L}$.

Corollaries [9]. The following statements hold true for any Lagrangian density of the type (32) in the sense of Definition 1:

1. If $\mathbf{f}_{s_{2}}^{2}$ alone acts on $\mathcal{L}$ leaving $\mathbf{v}$ arbitrary, from (38) we get Cauchy's balance of momentum

$$
\rho_{0} \ddot{\mathbf{x}}=\rho_{0} \mathbf{b}+\operatorname{Div} \mathbf{P},
$$

where $\mathbf{P}=-\partial_{\mathbf{F}} \mathcal{L} \in \operatorname{Hom}\left(T_{\mathbf{X}}^{*} \mathcal{B}_{0}, T_{\mathbf{x}}^{*} \mathcal{B}\right)$ is the first Piola-Kirchhoff stress and $\mathbf{b}=\partial_{\mathbf{x}} \mathcal{L} \in T_{\mathbf{x}}^{*} \mathcal{B}$ the vector of body forces. 
2. If $G$ arbitrary acts alone on $\mathcal{L}$, from (38) and the arbitrariness of the element $\xi$ of the Lie algebra of $G$ (chosen to define $\xi_{\mathcal{M}}(\boldsymbol{\nu})$ ) we get Capriz's balance of substructural interactions

$$
\rho_{0}\left(\frac{\cdot}{\partial_{\dot{\boldsymbol{\nu}}} \chi}-\partial_{\boldsymbol{\nu}} \chi\right)=-\mathbf{z}+\rho_{0} \boldsymbol{\beta}^{n i}+\operatorname{Div} \mathcal{S}
$$

in covariant way, where $\boldsymbol{\beta}^{n i}=-\rho_{0} \partial_{\boldsymbol{\nu}} w \in T_{\boldsymbol{\nu}}^{*} \mathcal{M}$ represents bulk noninertial external interactions acting on the substructure, $\mathcal{S}=-\partial_{\nabla \nu} \mathcal{L} \in$ Hom $\left(T_{\mathbf{X}}^{*} \mathcal{B}_{0}, T_{\nu}^{*} \mathcal{M}\right)$ takes into account contact substructural interactions between neighboring material elements (and is called microstress) and $\mathbf{z}=-\rho_{0} \partial_{\boldsymbol{\nu}} e \in T_{\boldsymbol{\nu}}^{*} \mathcal{M}$ indicates self-interactions of the substructure in each material element (and is called self-force).

3. Let $G=S O(3)$ and, for any element $\dot{\mathbf{q}} \wedge$ of its Lie algebra, $\mathbf{f}_{s_{3}}^{2}$ be such that $\mathbf{v}=\dot{\mathbf{q}} \wedge\left(\mathbf{x}-\mathbf{x}_{0}\right)$ with $\mathbf{x}_{0}$ a fixed point in space. In other words, we require that the same copy of $S O(3)$ acts both on the ambient space and on $\mathcal{M}$. If we require the invariance of $e$ with respect to $S O(3)$, we obtain

$$
\operatorname{skw}\left(\partial_{\mathbf{F}} e \mathbf{F}^{T}\right)=\mathrm{e}\left(\mathcal{A}^{T} \partial_{\boldsymbol{\nu}} e+\left(\nabla \mathcal{A}^{T}\right)^{t} \partial_{\nabla \boldsymbol{\nu}} e\right)
$$

where e is Ricci's alternator and $s k w(\cdot)$ extracts the skew-symmetric part of its argument. Previous statement render more perspicuous Remark 3 of $[9]$.

4. If $\mathbf{f}_{s_{1}}^{1}$ alone acts on $\mathcal{L}$, with $\mathbf{w}$ arbitrary, from 38 we get

$$
\overline{\left(\mathbf{F}^{T} \partial_{\dot{\mathbf{x}}} \mathcal{L}+\nabla \boldsymbol{\nu}^{T} \partial_{\dot{\boldsymbol{\nu}}} \mathcal{L}\right)}-\operatorname{Div}\left(\mathbb{P}-\left(\frac{1}{2} \rho_{0}|\dot{\mathbf{x}}|^{2}+\rho_{0} \chi(\boldsymbol{\nu}, \dot{\boldsymbol{\nu}})\right) \mathbf{I}\right)-\partial_{\mathbf{X}} \mathcal{L}=\mathbf{0}
$$

where $\mathbb{P}=\rho_{0} e \mathbf{I}-\mathbf{F}^{T} \mathbf{P}-\nabla \boldsymbol{\nu}^{T} \mathcal{S} \in A u t\left(T_{\mathbf{X}}^{*} \mathcal{B}_{0}\right)$, with $\mathbf{I}$ the second order unit tensor, is the modified Eshelby tensor for continua with substructure proposed in the general setting in [22], [23]. In particular, (43) coincides with the balance of configurational forces for a continuum with substructure in absence of dissipative internal forces driving defects. The balance (43), in fact, is only a consequence of the invariance with respect to relabeling.

5. Let $G=S O(3)$ and, for any element $\dot{\mathfrak{q}} \wedge$ of its Lie algebra, $\mathbf{f}_{s_{1}}^{1}$ is such that $\mathbf{w}=\dot{\mathfrak{q}} \wedge\left(\mathbf{X}-\mathbf{X}_{0}\right)$ with $\mathbf{X}_{0}$ a fixed point in $\mathcal{B}_{0}$. If the material is homogeneous, and only the special choices of $\mathbf{f}_{s_{2}}^{2}$ and $G$ just defined act on $\mathcal{L}, \mathbb{P}$ is symmetric.

Remark 9. It may be asked whether or not an integral version of (41) can be postulated as integral balance principle of substructural interactions and then used as a first principle. In general the answer is negative unless $\mathcal{M}$ is a linear space or is embedded in a linear space. The reason relies upon the circumstance that the eventual integrands $\boldsymbol{\beta}, \mathbf{z}$ and $\mathcal{S} \mathbf{n}$ would take values in $T^{*} \mathcal{M}$ which is a non-linear space unless the above mentioned situations of linearity for $\mathcal{M}$ occur, so the integrals are not defined. In other words, each time we use an integral version of (41) we presume implicitly an embedding in a linear space; on the contrary it does not make sense. 


\section{Discontinuity surfaces}

In common special cases, solutions of (33), (34) or, with other notations, of (40), (41) are not smooth and may display discontinuities concentrated on submanifolds of codimension 1. Moreover, experiments display domain formation and branching of microstructures of various nature (see e.g. cases of nematic order in liquid crystals, polarization in ferroelectrics, magnetization in micromagnetics, superconducting domains in superconductors etc.).

The presence of the gradient of the morphological descriptor in the list of entries of the energy accounts for the presence of interfaces of domain walls in a smeared sense. However, one may ask what happens when additional discontinuity surfaces occur and there is interaction between smeared and sharp interfaces. As an example, the us consider the solidification of a two-phase flow [24]. Two types of interfaces occur in this case. Interfaces between the two phases of the fluid and the interface between solid and fluid parts. The two-phase fluid is a complex body, the material element is a sort of box containing the two phases in different portions, so that a natural morphological descriptor is the volume or mass fraction $\omega$ of one phase with respect to the other. The presence of the gradient of $\omega$ in the list of entries of the energy allows us to account for diffuse interfaces between the two fluid phases. These diffuse interfaces interact with the interface solid-fluid. The latter may be described either either as a diffuse interface too or a sharp interface. The latter interpretation is a special case of the theory discussed below.

Really, even in single phase complex materials the energy may depend on the gradient of the order parameter to account for the inhomogeneous behavior of substructures. For example, in the case of micromorphic materials, each material element is a cell that may undergo micro-deformation independently of the neighboring cells, in addition to the participation to the overall macroscopic deformation [28]. The morphological descriptor is then a second-order symmetric tensor representing such an additional independent deformation. Such a situation may be representative of the behavior of elastomers, for example. Adjacent material elements may then undergo in principle different micro-deformations so that, in going from one element to the other, the energetic landscape changes in accord to the gradient of the micro-deformation and weakly non-local interaction effects of gradient type between neighboring material elements may be accounted for. Even in this case, additional discontinuity surfaces may occur as defects or shock and acceleration waves. Energetic effects associated with the gradient of the micro-deformation may influence them.

Analogous situation occurs also in quasicrystals where collective atomic modes (phason degrees of freedom) occur within each crystalline cell (the material element) and are represented by a stretchable vector. The energy depends both on the standard strain and the gradient of such a vector [31]. Even in this case, discontinuity surfaces such as racks or dislocations may intervene. Their behavior is influenced by the energetic effects associated 
with the gradient of the order parameter (here the vector mentioned above) but they are not strictly modeled through it.

The physical examples above allow us to clarify the point of view followed below. We treat, in fact, situations in which smeared interfaces or gradient effects due to inhomogeneous behavior of material substuctures interact with sharp interfaces or, more generally, discontinuity surfaces.

In particular, we focus our attention on a single discontinuity surface $\Sigma$ defined by

$$
\Sigma \equiv\{\mathbf{X} \in \operatorname{clB}, \quad f(\mathbf{X})=0\},
$$

with $f$ a smooth function with non-singular gradient. It is oriented by the normal vector field $\Sigma \ni \mathbf{X} \longmapsto \mathbf{m}(\mathbf{X})=\nabla f(\mathbf{X}) /|\nabla f(\mathbf{X})|$ and we use the notation $\Pi$ for $\mathbf{I}-\mathbf{m} \otimes \mathbf{m}$.

For any field $\tilde{e}(\cdot)$ continuous and piecewise continuously differentiable on $\Sigma$, we indicate with $\nabla_{\Sigma} e$ its surface gradient at $\mathbf{X}$, with $e=\tilde{e}(\mathbf{X})$, given by $\nabla_{\Sigma} e=\nabla e \Pi$. The opposite of the surface gradient of $\mathbf{m}$, namely $-\nabla_{\Sigma} \mathbf{m}$ is indicated with $\mathbf{L}$ and is the curvature tensor.

Let $\mathbf{X} \longmapsto a=\tilde{a}(\mathbf{X})$ be a generic field taking values in a linear space and suffering bounded discontinuities across $\Sigma$. For $\varepsilon>0$ we indicate with $a^{ \pm}$the $\operatorname{limits}_{\lim _{\varepsilon \rightarrow 0} a} a(\mathbf{X} \pm \varepsilon \mathbf{m})$ which are the outer $\left(a^{+}\right)$and inner $\left(a^{-}\right)$ traces of $a$ at $\Sigma$. Then we denote with $[a]=a^{+}-a^{-}$the jump of $a$ across $\Sigma$ and with $2\langle a\rangle=a^{+}+a^{-}$its average there, so that for any pair of fields $a_{1}$ and $a_{2}$ with the same properties of $a$ we have $\left[a_{1} a_{2}\right]=\left[a_{1}\right]\left\langle a_{2}\right\rangle+\left\langle a_{1}\right\rangle\left[a_{2}\right]$ if the product $a_{1} a_{2}$ is defined in some way and is distributive.

$[\mathbf{F}] \Pi=\mathbf{0}$ implies that $\Sigma$ is coherent. In other words the two pieces of the body separated by the surface do not suffer relative shear.

Moreover, if we attribute any 'virtual' motion to $\Sigma$ by means of a vector field $\Sigma \ni \mathbf{X} \longmapsto \mathbf{u}(\mathbf{X}) \in \mathbb{R}^{3}$ with normal component $U=\mathbf{u} \cdot \mathbf{m}$ and assume that the velocity $\dot{\mathbf{x}}$ may suffer bounded jumps across $\Sigma$, we get the condition $[\dot{\mathbf{x}}]=-U[\mathbf{F}] \mathbf{m}$, whose proof is textbook affairs.

As essential point we assume also that the order parameter map $\tilde{\boldsymbol{\nu}}$ is continuous across $\Sigma$. A special case in which such an assumption plays a prominent role is Landau's theory of phase transitions.

\subsection{The unstructured case}

We treat first the case in which $\Sigma$ is unstructured, i.e. it is free of own surface energy. We derive the balance equations across $\Sigma$ in a way that prove their covariance which has been not discussed so far. The procedure we adopt relies on the exploitation of an integral version of the pointwise relation (38).

We call part any regular subset $\mathfrak{b}$ of $\mathcal{B}_{0}$ with non vanishing volume measure. We consider an arbitrary part $\mathfrak{b}_{\Sigma}$ crossing $\Sigma$ in a way in which the intersection of its boundary $\partial \mathfrak{b}_{\Sigma}$ with $\Sigma$ be a curve and write for it the 
integral counterpart of (38), namely

$$
\frac{d}{d t} \int_{\mathfrak{b}_{\Sigma}} \mathcal{Q} d(\text { vol })+\int_{\partial \mathfrak{b}_{\Sigma}} \mathfrak{F} \cdot \mathbf{n} d(\text { area })=0 .
$$

If we take fixed the part $\mathfrak{b}_{\Sigma}$ with respect to the virtual motion of $\Sigma$, by the use of the transport and Gauss theorems (see, e.g., [27], [35]), as $\mathfrak{b}_{\Sigma} \rightarrow$ $\mathfrak{b}_{\Sigma} \cap \Sigma$ we find

$$
\begin{gathered}
\frac{d}{d t} \int_{\mathfrak{b}_{\Sigma}} \mathcal{Q} d(\text { vol }) \rightarrow-\int_{\mathfrak{b}_{\Sigma} \cap \Sigma}[\mathcal{Q}] U d(\text { vol }), \\
\int_{\partial \mathfrak{b}_{\Sigma}} \mathfrak{F} \cdot \mathbf{n} d(\text { area }) \rightarrow \int_{\mathfrak{b}_{\Sigma} \cap \Sigma}[\mathfrak{F}] \cdot \mathbf{m} d(\text { area }),
\end{gathered}
$$

so that the arbitrariness of $\mathfrak{b}_{\Sigma}$ implies the pointwise balance

$$
-[\mathcal{Q}] U+[\mathfrak{F}] \cdot \mathbf{m}=0
$$

Proposition 1 If the transformations A1, A2, A3 are smooth throughout the body, the validity of (48) and the invariance of $\mathcal{L}$ imply covariant pointwise interfacial balances across $\Sigma$ as in the list below.

1. The action of $\mathbf{f}_{s_{2}}^{2}$ alone implies the interfacial balance of standard interactions

$$
[\mathbf{P}] \mathbf{m}=-\rho_{0}[\dot{\mathbf{x}}] U
$$

2. The action of $G$ alone implies the interfacial balance of substructural interactions

$$
[\mathcal{S}] \mathbf{m}=-\rho_{0}\left[\partial_{\dot{\nu}} \chi\right] U
$$

3. The action of $\mathbf{f}_{s_{1}}^{1}$ alone implies the interfacial configurational balance along the normal $\mathbf{m}$ in absence of dissipative forces driving $\Sigma$, namely

$$
\mathbf{m} \cdot[\mathbb{P}] \mathbf{m}=\rho_{0} U\left[(\nabla \boldsymbol{\nu})^{*} \partial_{\dot{\boldsymbol{\nu}}} \chi\right] \cdot \mathbf{m}+\frac{1}{2} \rho_{0}[\chi(\boldsymbol{\nu}, \dot{\boldsymbol{\nu}})]-\frac{1}{2} \rho_{0} U^{2}\left[|\mathbf{F m}|^{2}\right]
$$

The proof is a consequence of direct calculation of (48). It is contained in the one of Theorem 2 below.

Balance equations at discontinuity unstructured surfaces in standard elasticity of simple bodies are derived by means of a direct evaluation of the variation of the total Lagrangian in [15]. 


\subsection{The structured case}

We consider here $\Sigma$ endowed with a surface energy density $\phi$ associated with surface tensions of standard and substructural nature.

Let $\mathbb{F}$ and $\mathbb{N}$ be defined by

$$
\mathbb{F}=\langle\mathbf{F}\rangle \Pi, \quad \mathbb{N}=\langle\nabla \boldsymbol{\nu}\rangle \Pi .
$$

They are surface deformation gradient and surface gradient of the order parameter at $\mathbf{X}$. There exist then two mappings $\tilde{\mathbb{F}}$ and $\tilde{\mathbb{N}}$ such that

$$
\begin{aligned}
& \Sigma \ni \mathbf{X} \stackrel{\tilde{\mathbb{F}}}{\longmapsto} \mathbb{F}=\tilde{\mathbb{F}}(\mathbf{X}) \in \operatorname{Hom}\left(T_{\mathbf{X}} \Sigma, T_{\mathbf{x}} \mathcal{B}\right), \\
& \Sigma \ni \mathbf{X} \stackrel{\widetilde{\mathbb{N}}}{\longmapsto} \mathbb{N}=\tilde{\mathbb{N}}(\mathbf{X}) \in \operatorname{Hom}\left(T_{\mathbf{X}} \Sigma, T_{\boldsymbol{\nu}} \mathcal{M}\right) .
\end{aligned}
$$

Elementary algebra provides us

$$
\begin{gathered}
\langle\mathbf{F}\rangle=\mathbb{F}+(\langle\mathbf{F}\rangle \mathbf{m}) \otimes \mathbf{m}, \\
\langle\nabla \boldsymbol{\nu}\rangle=\mathbb{N}+(\langle\nabla \boldsymbol{\nu}\rangle \mathbf{m}) \otimes \mathbf{m} .
\end{gathered}
$$

The surface energy density is then defined by

$$
(\mathbf{m}, \mathbb{F}, \boldsymbol{\nu}, \mathbb{N}) \stackrel{\tilde{\phi}}{\longmapsto} \phi=\tilde{\phi}(\mathbf{m}, \mathbb{F}, \boldsymbol{\nu}, \mathbb{N})
$$

and we assume that $\tilde{\phi}$ be sufficiently smooth. It is worth noting that the presence of the normal $\mathbf{m}$ in the list of entries of $\tilde{\phi}$ points out that we are considering anisotropic surfaces.

We require the invariance of $\phi$ with respect (i) to general changes of observers and (ii) to relabeling of $\Sigma$. As discussed above, changes of observers are characterized by the action of the group of automorphisms of $\mathcal{E}^{3}$ and of a generic Lie group over $\mathcal{M}$. However, definition A3 of $\mathbf{f}_{s_{1}}^{1}$ needs to be modified in order to describe the relabeling of $\Sigma$ in addition to the overall relabeling of $\mathcal{B}_{0}$. Instead of $s_{1} \longmapsto \mathbf{f}_{s_{1}}^{1}$ we should consider time-parametrized families $s_{1} \longmapsto \hat{\mathbf{f}}_{s_{1}}^{1}$ of elements of $\operatorname{SDiff}\left(\mathcal{B}_{0}\right)$ characterized by the properties listed below.

Relabeling of $\mathcal{B}_{0}$ including $\Sigma$.

1. The map $s_{1} \longmapsto \hat{\mathbf{f}}_{s_{1}}^{1}$ satisfies A1. Moreover, we require that the field $\mathcal{B}_{0} \ni \mathbf{X} \longmapsto \mathbf{w}=\tilde{\mathbf{w}}(\mathbf{X})=\hat{\mathbf{f}}_{0}^{1^{\prime}}(\mathbf{X})$ is at least of class $C^{1}\left(\mathcal{B}_{0}\right)$, then also across and along $\Sigma$.

2. Each $\hat{\mathbf{f}}_{s_{1}}^{1}$ preserves the elements of area of $\Sigma$. Namely, if $d A$ is the element of area of $\Sigma$ in $\mathcal{B}_{0}, d A=\hat{\mathbf{f}}_{s_{1}}^{1 *} \circ d A$, where the asterisk indicates push forward.

3. $(\nabla \mathbf{w}) \mathbf{m}=\mathbf{0}$.

4. $\nabla_{\Sigma} w_{m}=0$, with $w_{m}=\mathbf{w} \cdot \mathbf{m}$. 
Two lemmas are useful for subsequent calculations.

Lemma 1. For any isocoric vector field $\tilde{\mathbf{w}}(\cdot)$ of class $C^{1}\left(\mathcal{B}_{0}\right)$,

$$
\Pi \cdot \nabla_{\Sigma} \mathbf{w}=((\nabla \mathbf{w}) \mathbf{m}) \cdot \mathbf{m}
$$

with $\mathbf{w}=\tilde{\mathbf{w}}(\mathbf{X})$.

The result follows from direct calculation.

A second order tensor field $\Sigma \ni \mathbf{X} \stackrel{\tilde{\mathbf{A}}}{\longmapsto} \mathbf{A}=\tilde{\mathbf{A}}(\mathbf{X}) \in \operatorname{Hom}\left(\mathbb{R}^{3}, \mathbb{R}^{3}\right)$ is called superficial if $\mathbf{A m}=\mathbf{0}$ at each $\mathbf{X}$.

Lemma 2. For any second-order superficial tensor field $\tilde{\mathbf{A}}$ on $\Sigma$, one gets

$$
\mathbf{m} \cdot \operatorname{Div}_{\Sigma} \mathbf{A}=\mathbf{A} \cdot \mathrm{L}
$$

Definition 2. A surface energy density $\phi$ is invariant with respect to the action of $\hat{\mathbf{f}}_{s_{1}}^{1}, \mathbf{f}_{s_{2}}^{2}$ and $G$ if

$$
\tilde{\phi}(\mathbf{m}, \mathbb{F}, \boldsymbol{\nu}, \mathbb{N})=\tilde{\phi}\left(\nabla \hat{\mathbf{f}}^{1 T} \mathbf{m},\left(\operatorname{grad}_{\Sigma} \mathbf{f}^{2}\right) \mathbb{F}\left(\nabla \hat{\mathbf{f}}^{1}\right)^{-1}, \boldsymbol{\nu}_{g}, \mathbb{N}_{g}\left(\nabla \hat{\mathbf{f}}^{1}\right)^{-1}\right)
$$

for any $g \in G$ and $s_{1}, s_{2} \in \mathbb{R}^{+}$, where $\mathbb{N}_{g}=\left\langle\nabla \boldsymbol{\nu}_{g}\right\rangle \Pi$ and we have used notations common to Definition 1.

Let $\mathfrak{X}$ be a sufficiently smooth vector density defined over $\Sigma$ by $\mathfrak{X}=-\phi \Pi \mathbf{w}+\left(\partial_{\mathbb{F}} \phi\right)^{T}(\mathbf{v}-\langle\mathbf{F}\rangle \mathbf{w})+\left(\partial_{\mathbb{N}} \phi\right)^{T}\left(\xi_{\mathcal{M}}(\boldsymbol{\nu})-\langle\nabla \boldsymbol{\nu}\rangle \mathbf{w}\right)-\left(\partial_{\mathbf{m}} \phi \otimes \mathbf{m}\right) \mathbf{w}$.

It is the counterpart of $\mathfrak{F}$ for a surface.

Theorem 2. Let $\Sigma$ be a structured surface with surface energy $\phi$. Let us assume

$$
\frac{d}{d t} \int_{\mathfrak{b}_{\Sigma}} \mathcal{Q} d(v o l)+\int_{\partial \mathfrak{b}_{\Sigma}} \mathfrak{F} \cdot \mathbf{n} d(\text { area })+\int_{\partial\left(\mathfrak{b}_{\Sigma} \cap \Sigma\right)} \mathfrak{X} \cdot \mathrm{nd}(\text { length })=0
$$

for any part $\mathfrak{b}_{\Sigma}$ of $\mathcal{B}_{0}$ crossing $\Sigma$. If $\mathcal{L}$ and $\phi$ are invariant with respect to $\hat{\mathbf{f}}_{s_{1}}^{1}, \mathbf{f}_{s_{2}}^{2}$ and $G$, covariant pointwise balances across $\Sigma$ follow as in the list below.

1. The action of $\mathbf{f}_{s_{2}}^{2}$ alone implies the interfacial balance of standard interactions

$$
[\mathbf{P}] \mathbf{m}+\operatorname{Div}_{\Sigma} \mathbb{T}=-\rho_{0}[\dot{\mathbf{x}}] U
$$

where $\mathbb{T}=-\partial_{\mathbb{F}} \phi \in H o m\left(T_{\mathbf{X}} \Sigma, T_{\mathbf{x}}^{*} \mathcal{B}\right)$ is the surface Piola-Kirchhoff stress. 
2. The action of $G$ alone implies the interfacial balance of substructural interactions

$$
[\mathcal{S}] \mathbf{m}+\operatorname{Div}_{\Sigma} \mathbb{S}-\mathfrak{z}=-\rho_{0}\left[\partial_{\dot{\boldsymbol{\nu}}} \chi\right] U,
$$

where $\mathbb{S}=-\partial_{\mathbb{N}} \phi \in \operatorname{Hom}\left(T_{\mathbf{X}} \Sigma, T_{\nu}^{*} \mathcal{M}\right)$ is the surface microstress and $\mathfrak{z}=\partial_{\nu} \phi \in T_{\nu}^{*} \mathcal{M}$ the surface self-force.

3. The action of $\hat{\mathbf{f}}_{s_{1}}^{1}$ alone implies the interfacial configurational balance along the normal $\mathbf{m}$ in absence of dissipative forces driving $\Sigma$, namely

$$
\begin{gathered}
\mathbf{m} \cdot[\mathbb{P}] \mathbf{m}+\mathbb{C}_{\tan } \cdot \mathbf{L}+\operatorname{Div}_{\Sigma} \mathfrak{c}= \\
=\rho_{0} U\left[(\nabla \boldsymbol{\nu})^{*} \partial_{\dot{\boldsymbol{\nu}}} \chi\right] \cdot \mathbf{m}+\rho_{0}[\chi(\boldsymbol{\nu}, \dot{\boldsymbol{\nu}})]-\frac{1}{2} \rho_{0} U^{2}\left[|\mathbf{F m}|^{2}\right],
\end{gathered}
$$

where

$$
\mathbb{C}_{\mathrm{tan}}=\phi \Pi-\mathbb{F}^{T} \mathbb{T}-\mathbb{N}^{T} \mathbb{S}
$$

is a generalized version of the surface Eshelby stress and

$$
\mathfrak{c}=-\partial_{\mathbf{m}} \phi-\mathbb{T}^{T}\langle\mathbf{F}\rangle \mathbf{m}-\mathbb{S}^{T}\langle\nabla \boldsymbol{\nu}\rangle \mathbf{m}
$$

is a surface shear.

By following different procedures, equation (63) has been derived in [17] while equations (64) and (65) in [22], [23] (the version of 65) for simple bodies is clearly obtained in [16]). Here, with Theorem 2 we prove their covariance: this is the main novelty of the theorem itself.

\subsection{Proof of Theorem 2}

Step 1.

In accord to Definition 2, since $\tilde{\phi}$ is invariant, we have

$$
\left.\frac{d}{d s_{1}} \tilde{\phi}\left(\nabla \hat{\mathbf{f}}^{1 T} \mathbf{m},\left(\operatorname{grad}_{\Sigma} \mathbf{f}^{2}\right) \mathbb{F}\left(\nabla \hat{\mathbf{f}}^{1}\right)^{-1}, \boldsymbol{\nu}_{g}, \mathbb{N}_{g}\left(\nabla \hat{\mathbf{f}}^{1}\right)^{-1}\right)\right|_{s_{1}=0, s_{2}=0, s_{3}=0}=0,
$$

$\left.\frac{d}{d s_{2}} \tilde{\phi}\left(\nabla \hat{\mathbf{f}}^{1 T} \mathbf{m},\left(\operatorname{grad}_{\Sigma} \mathbf{f}^{2}\right) \mathbb{F}\left(\nabla \hat{\mathbf{f}}^{1}\right)^{-1}, \boldsymbol{\nu}_{g}, \mathbb{N}_{g}\left(\nabla \hat{\mathbf{f}}^{1}\right)^{-1}\right)\right|_{s_{1}=0, s_{2}=0, s_{3}=0}=0$

$\left.\frac{d}{d s_{3}} \tilde{\phi}\left(\nabla \hat{\mathbf{f}}^{1 T} \mathbf{m},\left(\operatorname{grad}_{\Sigma} \mathbf{f}^{2}\right) \mathbb{F}\left(\nabla \hat{\mathbf{f}}^{1}\right)^{-1}, \boldsymbol{\nu}_{g}, \mathbb{N}_{g}\left(\nabla \hat{\mathbf{f}}^{1}\right)^{-1}\right)\right|_{s_{1}=0, s_{2}=0, s_{3}=0}=0$

which correspond respectively to

$$
\mathbb{F}^{T} \mathbb{T} \cdot \nabla_{\Sigma} \mathbf{w}+\mathbb{N}^{T} \mathbb{S} \cdot \nabla_{\Sigma} \mathbf{w}+\partial_{\mathbf{m}} \phi \cdot(\nabla \mathbf{w}) \mathbf{m}=0,
$$




$$
\begin{gathered}
\mathbb{T} \cdot \nabla_{\Sigma} \mathbf{v}=0, \\
\mathfrak{z} \cdot \xi_{\mathcal{M}}(\boldsymbol{\nu})+\mathbb{S} \cdot \nabla_{\Sigma} \xi_{\mathcal{M}}(\boldsymbol{\nu})=0 .
\end{gathered}
$$

They will be useful tools below.

Step 2.

If we shrink $\mathfrak{b}_{\Sigma}$ to $\mathfrak{b}_{\Sigma} \cap \Sigma$ uniformly in time, transport and Gauss theorems (see also (46) and (47)) allow us to obtain the pointwise balance

$$
-[\mathcal{Q}] U+[\mathfrak{F}] \cdot \mathbf{m}+\operatorname{Div}_{\Sigma} \mathfrak{X}=0,
$$

thanks to the arbitrariness of $\mathfrak{b}_{\Sigma}$. Of course, the sole difference between (74) and (48) is the term $\operatorname{Div}_{\Sigma} \mathfrak{X}$ accounting for the interfacial structure of the surface $\Sigma$.

Step 3. Deduction of the referential interfacial balance of standard interactions (63).

If $\mathbf{f}^{2}$ acts alone, then

$$
\mathfrak{X}=\mathbb{T}^{T} \mathbf{v}, \quad \mathcal{Q}=\rho \dot{\mathbf{x}} \cdot \mathbf{v}, \quad \mathfrak{F}=-\mathbf{P}^{T} \mathbf{v} .
$$

Moreover, thanks to (72) we get

$$
\operatorname{Div}_{\Sigma} \mathfrak{X}=\mathbf{v} \cdot \operatorname{Div}_{\Sigma} \mathbb{T} .
$$

Then, from (74) we obtain (63) thanks to the arbitrariness of $\mathbf{v}$, which is continuous across $\Sigma$.

Step 4. Deduction of the referential interfacial balance of substructural interactions (64).

If $G$ acts alone, then

$$
\mathfrak{X}=\mathbb{S}^{T} \xi_{\mathcal{M}}(\boldsymbol{\nu}), \quad \mathcal{Q}=\rho_{0} \partial_{\dot{\boldsymbol{\nu}}} \chi \cdot \xi_{\mathcal{M}}(\boldsymbol{\nu}), \quad \mathfrak{F}=-\mathcal{S}^{T} \xi_{\mathcal{M}}(\boldsymbol{\nu}) .
$$

Moreover, thanks to (73) we get

$$
\operatorname{Div}_{\Sigma} \mathfrak{X}=\xi_{\mathcal{M}}(\boldsymbol{\nu}) \cdot\left(\operatorname{Div}_{\Sigma} \mathbb{S}-\mathfrak{z}\right) .
$$

Then, from (744) we obtain (64) thanks to the arbitrariness of the element $\xi$ selected in the Lie algebra of $G$.

Step 5. Deduction of the balance of configurational forces along the normal $\mathbf{m}$ (i.e. (65)).

If $\hat{\mathbf{f}}^{1}$ acts alone, then

$$
\mathcal{Q}=-\rho \mathbf{F}^{T} \dot{\mathbf{x}} \cdot \mathbf{w}-\rho_{0}(\nabla \boldsymbol{\nu})^{*} \partial_{\dot{\boldsymbol{\nu}}} \chi \cdot \mathbf{w},
$$




$$
\mathfrak{F}=\left(\left(\frac{1}{2} \rho_{0}|\dot{\mathbf{x}}|^{2}+\rho_{0} \chi(\boldsymbol{\nu}, \dot{\boldsymbol{\nu}})\right) \mathbf{I}-\mathbb{P}\right) \mathbf{w}
$$

and, after some algebra,

$$
\mathfrak{X}=-\mathbb{C}_{\tan }^{T} \mathbf{w}-\mathfrak{c} w_{m},
$$

with $\mathbb{C}_{\text {tan }}$ and $\mathfrak{c}$ defined respectively by (66) and (67) and $w_{m}=\mathbf{w} \cdot \mathbf{m}$.

Now, equation (74) comes into play: we will evaluate the component along $\mathbf{m}$ of the right-hand side term of (74), a vector, by taking also into account the arbitrariness of $\mathbf{w}$.

First we write

$$
\begin{gathered}
-[\mathcal{Q}] U+[\mathfrak{F}] \cdot \mathbf{m}=\rho_{0}\left[\mathbf{F}^{T} \dot{\mathbf{x}}\right] U \cdot \mathbf{w}+\rho_{0}\left[(\nabla \boldsymbol{\nu})^{*} \partial_{\dot{\boldsymbol{\nu}}} \chi\right] U \cdot \mathbf{w}+ \\
+\frac{1}{2} \rho_{0}\left[|\dot{\mathbf{x}}|^{2}\right] \mathbf{w} \cdot \mathbf{m}+\rho_{0}[\chi(\boldsymbol{\nu}, \dot{\boldsymbol{\nu}})] \mathbf{w} \cdot \mathbf{m}-[\mathbb{P}] \mathbf{w} \cdot \mathbf{m} .
\end{gathered}
$$

Moreover, by using (71) and Lemma 1, we also get

$$
\operatorname{Div}_{\Sigma}\left(\mathbb{C}_{\tan }^{T} \mathbf{w}+\mathfrak{c} w_{m}\right)=\mathbf{w} \cdot\left(\operatorname{Div}_{\Sigma} \mathbb{C}_{\tan }+\left(\operatorname{Div}_{\Sigma} \mathfrak{c}\right) \mathbf{m}\right) .
$$

Of course, in obtaining (83), properties 3 and 4 of the definition of the relabeling $\hat{\mathbf{f}}^{1}$ of $\mathcal{B}_{0}$ including $\Sigma$ play a crucial role.

By inserting (82) and (83) in (174), thanks to the arbitrariness of $\mathbf{w}$, we obtain

$$
\begin{gathered}
\rho_{0}\left[\mathbf{F}^{T} \dot{\mathbf{x}}\right] U+\rho_{0}\left[(\nabla \boldsymbol{\nu})^{*} \partial_{\dot{\boldsymbol{\nu}} \chi} \chi U+\frac{1}{2} \rho_{0}\left[|\dot{\mathbf{x}}|^{2}\right] \mathbf{m}+\right. \\
+\rho_{0}[\chi(\boldsymbol{\nu}, \dot{\boldsymbol{\nu}})] \mathbf{m}=\left[\mathbb{P}^{T}\right] \mathbf{m}+\operatorname{Div}_{\Sigma} \mathbb{C}_{\tan }+\left(\operatorname{Div}_{\Sigma} \mathfrak{c}\right) \mathbf{m}
\end{gathered}
$$

and we shall evaluate the component along $\mathbf{m}$ of (84).

First we focus our attention on terms involving $\dot{\mathrm{x}}$ and $\dot{\boldsymbol{\nu}}$. Let us introduce the averaged velocity $\overline{\mathbf{v}}$ given by

$$
\overline{\mathbf{v}}=\langle\dot{\mathbf{x}}\rangle+U\langle\mathbf{F}\rangle \mathbf{m} .
$$

With the help of the relation $[\dot{\mathbf{x}}]=-U[\mathbf{F}] \mathbf{m}$ introduced previously, we then get

$$
\rho_{0}\left[\mathbf{F}^{T} \dot{\mathbf{x}}\right] U \cdot \mathbf{m}=\rho_{0}[\dot{\mathbf{x}}] \cdot \overline{\mathbf{v}}-\rho_{0}\left[|\dot{\mathbf{x}}|^{2}\right]
$$

in other words the normal component of the vector $\rho_{0}\left[\mathbf{F}^{T} \dot{\mathbf{x}}\right] U$ is equal to minus the jump of the relative kinetic energy $\frac{1}{2}\left[\rho_{0}|\dot{\mathbf{x}}-\overline{\mathbf{v}}|^{2}\right]$ as it is simple to verify.

Still taking into account the relation $[\dot{\mathbf{x}}]=-U[\mathbf{F}] \mathbf{m}$ and the definition of $\overline{\mathbf{v}}$, we also find

$$
\frac{1}{2} \rho_{0}\left[|\dot{\mathbf{x}}|^{2}\right]=-\rho_{0}[\dot{\mathbf{x}}] \cdot \overline{\mathbf{v}}+\frac{1}{2} U^{2}\left[|\mathbf{F m}|^{2}\right] .
$$

Now, by evaluating the normal component of (84), using (86), (87) and taking into account that $\mathbf{m} \cdot \operatorname{Div}_{\Sigma} \mathbb{C}_{\tan }=\mathbb{C}_{\tan } \cdot \mathrm{L}$ as a consequence of Lemma 2 , we obtain (65) and the theorem is proven. 


\section{References}

1. Abraham, R., Marsden, J. E., Foundations of mechanics. The Benjamin/Cummings Publishing Company, London, 1978.

2. Brezis, H., Li, Y. (2001), Topology and Sobolev spaces, J. Funct. Anal., 183, 321-369.

3. Capriz, G. (1985), Continua with latent microstructure, Arch. Rational Mech. Anal., 90, 43-56.

4. Capriz, G., Continua with microstructure. Springer-Verlag, Berlin, 1989.

5. Capriz, G. (2001), Continua with substructure. Part I and Part II, Phys. Mesomech., 3, 5-14 and 37-50.

6. Capriz, G. (2003), Elementary preamble to a theory of granular gases, Rend. Sem. Mat. Univ. Padova, 110, 179-198.

7. Capriz, G., Giovine, P. (1997), On microstructural inertia, Math. Mod. meth. Appl. Sci., 7, 211-216.

8. Capriz, G., Mariano, P. M. (2003), Balance at a junction in coherent interfaces in continua with substructure, in Advances in multifield theoris for continua with substructure, G. Capriz and P. M. Mariano Edts, Birkhauser, Boston, 243-263.

9. Capriz, G., Mariano, P. M. (2003), Symmetries and Hamiltonian formalism for complex materials, J. Elasticity, 72, 57-70.

10. Cosserat, E., Cosserat, F., Sur la théorie des corps deformables, Dunod, Paris, 1909.

11. de Gennes, P.G., Prost, J., The physics of liquid crystals, Claredon Press (1993).

12. Del Piero, G., Owen, D. R. (1993), Structured deformation of continua, Arch. Rational Mech. Anal., 124, 99-155.

13. Del Piero, G., Owen, D. R., Structured deformation, Quaderni dell'Istituto di Alta Matematica, Florence, 2000.

14. Ericksen, J. L. (1991), Liquid crystals with variable degree of orientation, Arch. Rational Mech. Anal., 113, 97-120.

15. Fatecau, R. C., Marsden, J. E., West, M. (2003), Variational multisymplectic formulation of non-smooth continuum mechanics, in Perspective and problems in nonlinear sciences, E. Kaplan, J. E. Marsden, K. R. Sreenivasan, Springer, Berlin, 229-261.

16. Gurtin, M. E. (1995), The nature of configurational forces, Arch. Rational Mech. Anal., 131, 67-100.

17. Gurtin, M. E., Struthers, A. (1990), Multiphase thermomechanics with interfacial structure. III. Evolving phase boundaries in the presence of bulk deformation, Arch. Rational Mech. Anal., 112, 97-160.

18. Hang, F., Lin, F. (2003), Topology of Sobolev mappings III, Comm. Pure Appl. Math., 61, 1383-1415.

19. Helgason, S., Differential geometry Lie groups and symmetric spaces. Academic Press, New York, 1978.

20. Leslie, F. M. (1968), Some constitutive equations for liquid crystals, Arch. Rational Mech. Anal., 28, 265-283. 
21. Mariano, P. M. (1999), Some remarks on the variational description of microcracked bodies, Int. J. Non-Linear Mech., 34, 633-642.

22. Mariano, P. M. (2000), Configurational forces in continua with microstructure, Z. angew. Math. Phys. ZAMP, 51, 752-791.

23. Mariano, P. M. (2001), Multifield theories in mechanics of solids, Adv. Appl. Mech., 38, 1-93.

24. Mariano, P. M. (2004), Some thermodynamical aspects of the solidification of two-phase flows, Meccanica, 39, 369-382.

25. Mariano, P. M., Elements of multifield theories of complex bodies, Birkhauser, Boston, in preparation.

26. Mariano, P. M., Stazi, F. L. (2004) Strain localization due to crackmicrocrack interaction: X-FEM for a multifield approach, Comp. Meth. Appl. Mech. Eng., in print.

27. Marsden, J. E., Hughes, T. R. J., Mathematical foundations of elasticity. Prentice-Hall, New Jersey, 1983.

28. Mindlin, R. D. (1964), Micro-structures in linear elasticity, Arch. Rational Mech. Anal., 16, 51-78.

29. Noll, W. (1958), A mathematical theory of the mechanical behavior of continuous media. Arch. Rational Mech. Anal., 2, 197-226.

30. Noll, W. (1972), A new mathematical theory of simple materials. Arch. Rational Mech. Anal., 48, 1-50.

31. Rochal, S. B., Lorman, V. L. (2001), Minimal model of the phononphason dynamics in icosahedral quasicrystls and its application to the problem of internal friction in the $i$-AlPdMn alloy, Phys. Rev. B, 66, 144204 1-9.

32. Segev, R. (1994), A geometrical framework for the statics of materials with microstructure, Mat. Models Methods Appl. Sci., 4, 871-897.

33. Sharpe, R. W., Differential geometry. Springer-Verlag, Berlin, 1997.

34. Šilhavý, M. (1985), Phase transitions in non-simple bodies, Arch. Rational Mech. Anal., 88, 135-161.

35. Šilhavý, M., The mechanics and thermodynamics of continuous media, Springer, Berlin, 1997. 